\title{
SNS Sample Activation Calculator Flux Recommendations and Validation
}

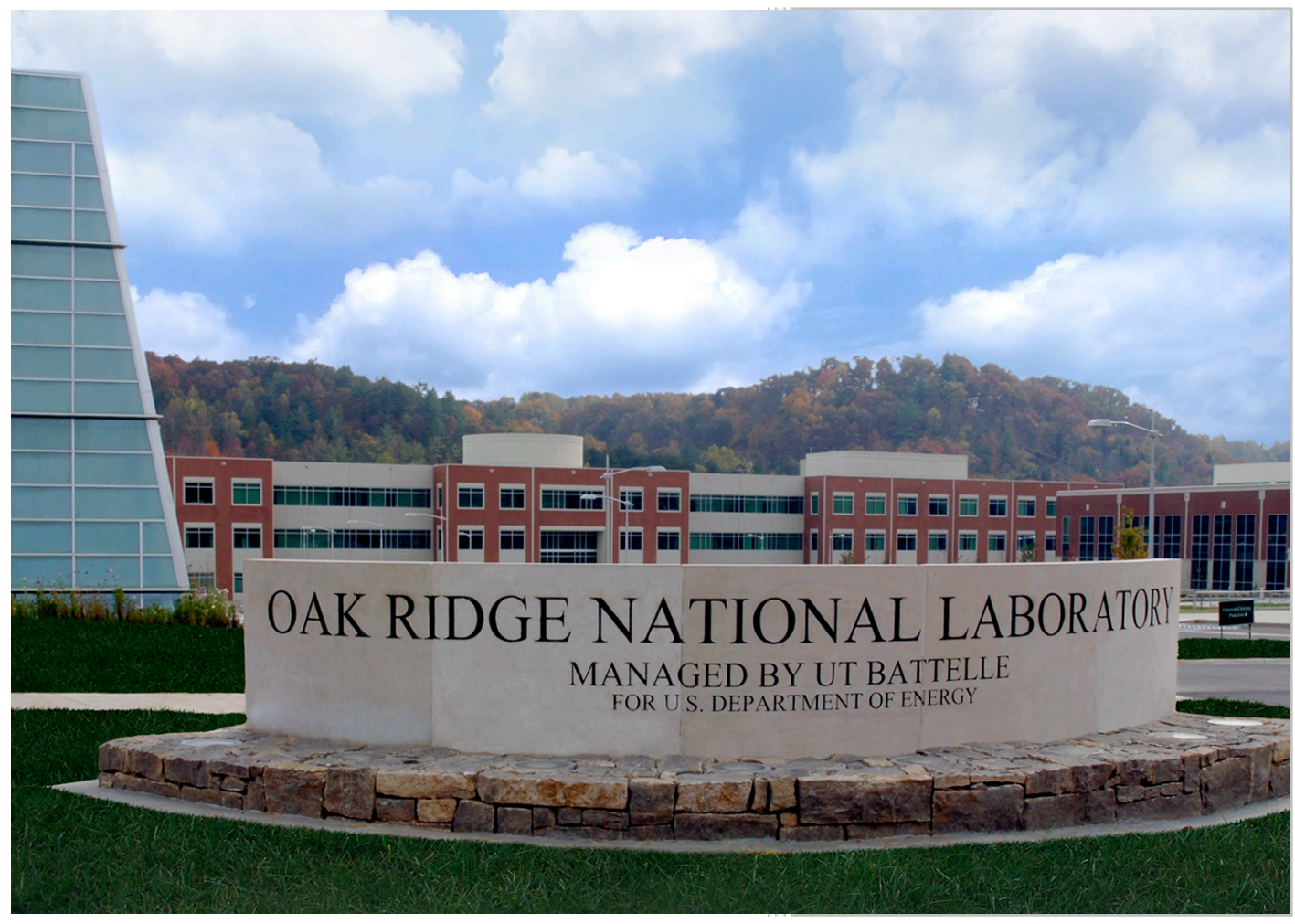

Approved for public release; distribution is unlimited.
T.C. McClanahan

F.X. Gallmeier

E.B. Iverson

W. Lu

February 2015 


\section{DOCUMENT AVAILABILITY}

Reports produced after January 1, 1996, are generally available free via US Department of Energy (DOE) SciTech Connect.

Website http://www.osti.gov/scitech/

Reports produced before January 1, 1996, may be purchased by members of the public from the following source:

National Technical Information Service

5285 Port Royal Road

Springfield, VA 22161

Telephone 703-605-6000 (1-800-553-6847)

TDD 703-487-4639

Fax 703-605-6900

E-mail info@ntis.gov

Website http://www.ntis.gov/help/ordermethods.aspx

Reports are available to DOE employees, DOE contractors, Energy Technology Data Exchange representatives, and International Nuclear Information System representatives from the following source:

Office of Scientific and Technical Information

PO Box 62

Oak Ridge, TN 37831

Telephone 865-576-8401

Fax 865-576-5728

E-mail reports@osti.gov

Website http://www.osti.gov/contact.html

This report was prepared as an account of work sponsored by an agency of the United States Government. Neither the United States Government nor any agency thereof, nor any of their employees, makes any warranty, express or implied, or assumes any legal liability or responsibility for the accuracy, completeness, or usefulness of any information, apparatus, product, or process disclosed, or represents that its use would not infringe privately owned rights. Reference herein to any specific commercial product, process, or service by trade name, trademark, manufacturer, or otherwise, does not necessarily constitute or imply its endorsement, recommendation, or favoring by the United States Government or any agency thereof. The views and opinions of authors expressed herein do not necessarily state or reflect those of the United States Government or any agency thereof. 


\title{
SNS SAMPLE ACTIVATION CALCULATOR VALIDATION
}

\author{
T.C. McClanahan \\ F.X. Gallmeier \\ E.B. Iverson \\ $\mathrm{W} . \mathrm{Lu}$
}

Date Published: February 2015

\author{
Prepared by \\ OAK RIDGE NATIONAL LABORATORY \\ Oak Ridge, Tennessee 37831-6283 \\ managed by \\ UT-BATTELLE, LLC \\ for the \\ US DEPARTMENT OF ENERGY \\ under contract DE-AC05-00OR22725
}




\section{CONTENTS}

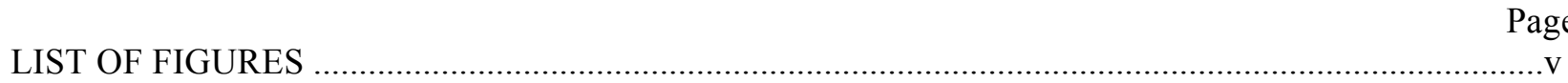

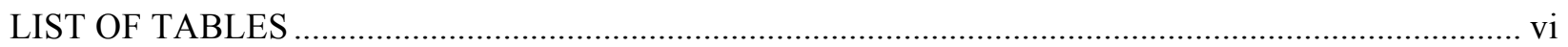

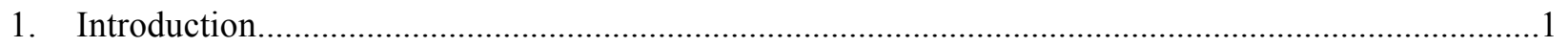

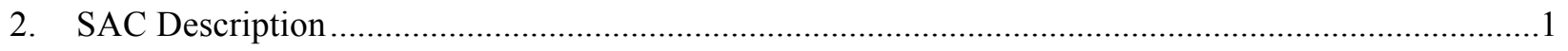

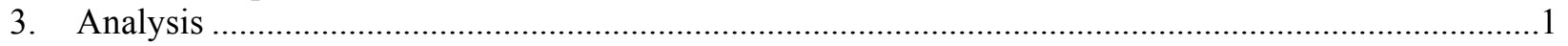

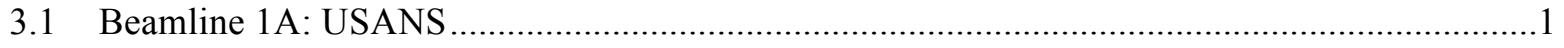

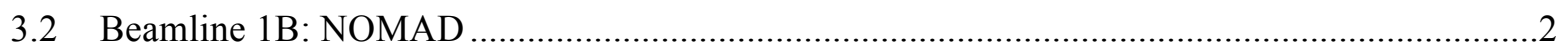

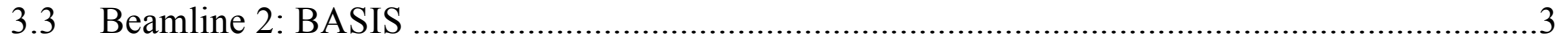

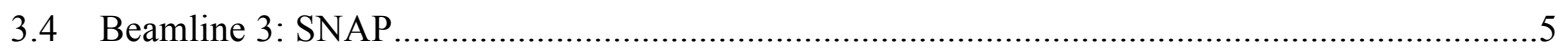

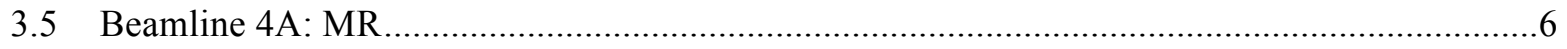

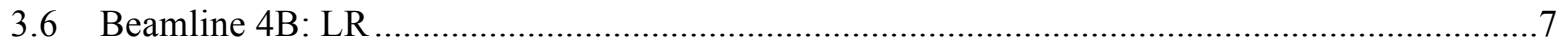

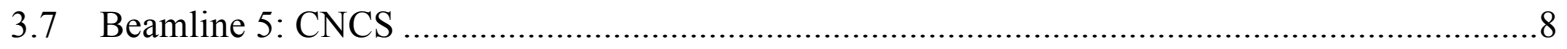

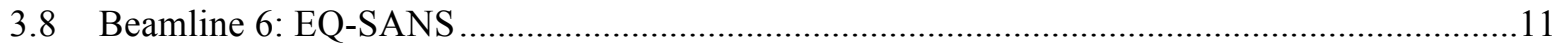

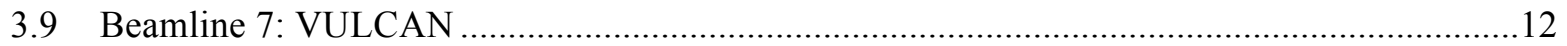

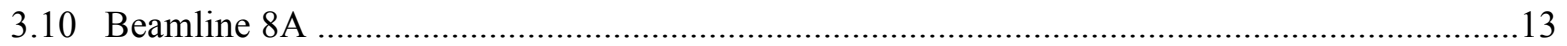

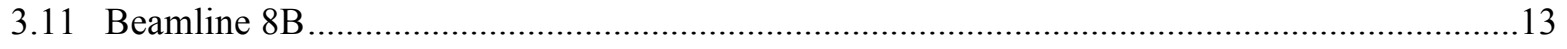

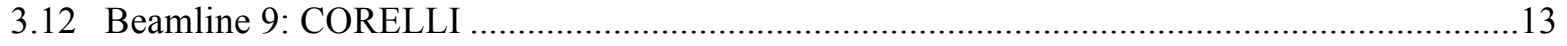

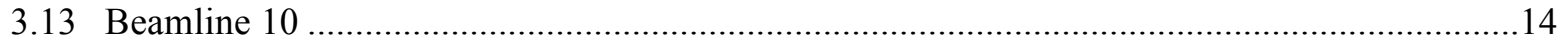

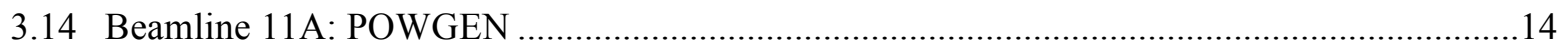

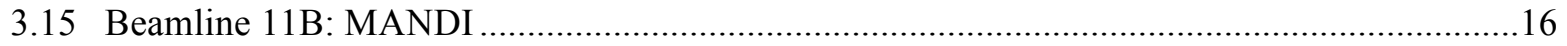

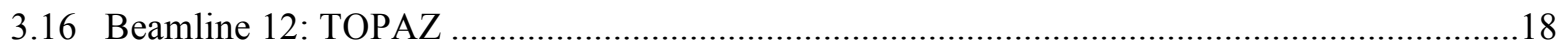

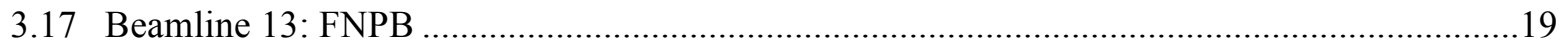

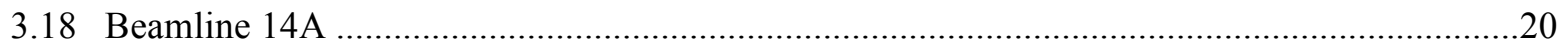

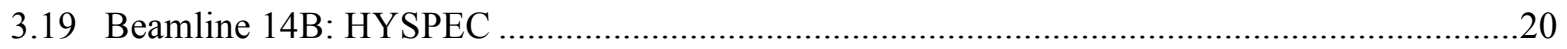

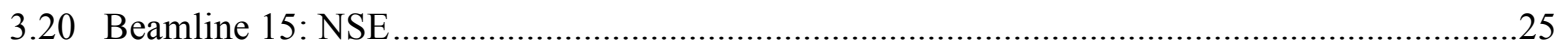

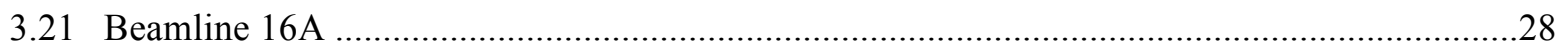

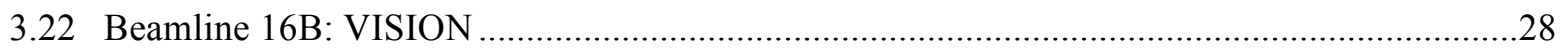

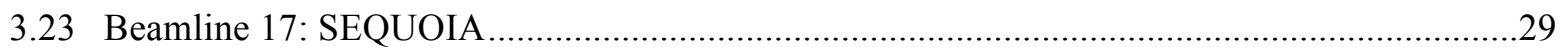

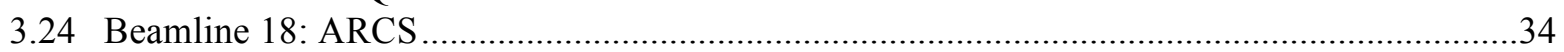

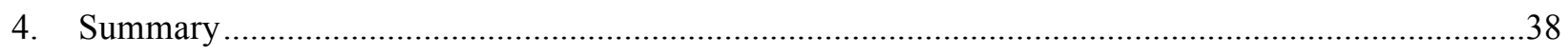

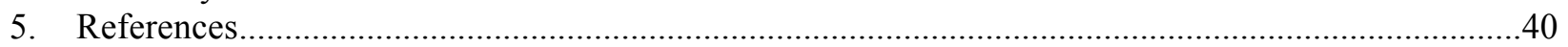




\section{LIST OF FIGURES}

Figure

Fig. 1. NOMAD Neutron Flux Comparison

Fig. 2. Comparison of Simulated NOMAD Neutron Fluxes by Mcstas/Gallmeier [5, 6] and

IDEAS/Neuefeind (from Joerg) [4]

Fig. 3. Comparison of measured (red) and simulated (green) NOMAD neutron current by

Neuefeind et al [4]

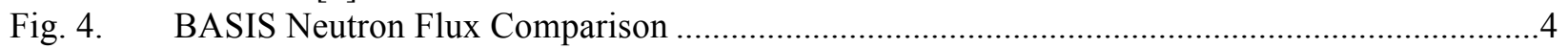

Fig. 5. BASIS Neutron Flux as Measured by Mamontov [7] .........................................................

Fig. 6. SNAP Wavelength Flux Comparison ...........................................................................6

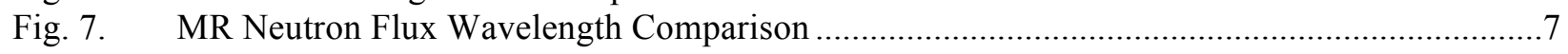

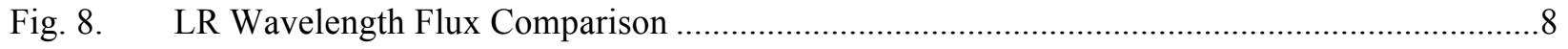

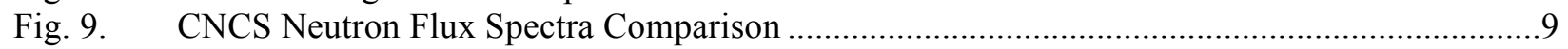

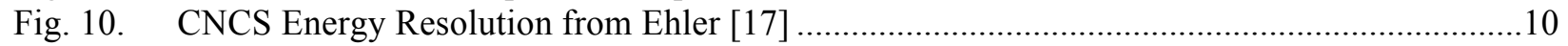

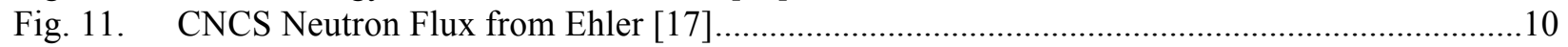

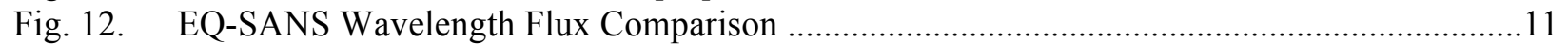

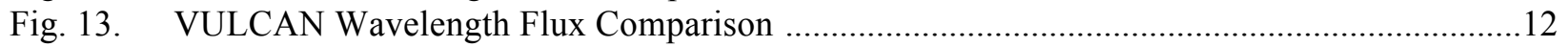

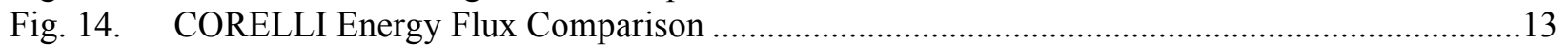

Fig. 15. POWGEN High Intensity Energy Flux Comparison .......................................................15

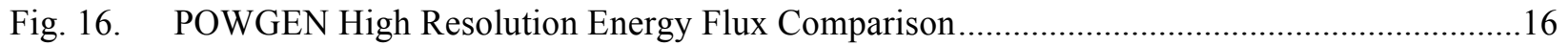

Fig. 17. MANDI High Intensity Energy Flux Comparison................................................................17

Fig. 18. TOPAZ High Intensity Energy Flux Comparison ..............................................................19

Fig. 19. FNPB Neutron Flux Spectra Comparison at $15 \mathrm{~cm}$ Distance From the Neutron Guide

Exit. 20

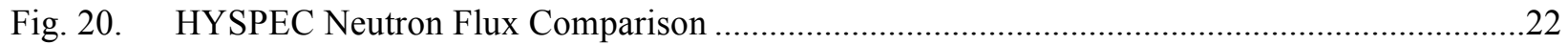

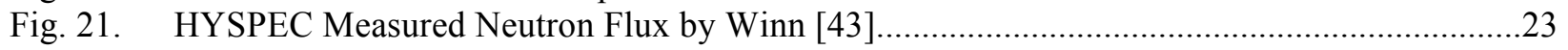

Fig. 22. HYSPEC Measured Energy Resolution by Winn [43] .....................................................23

Fig. 23. HYSPEC Measured Neutron Flux as reported by Stone [20] ................................................24

Fig. 24. HYPSEC Simulated Energy Flux and Energy Resolution calculated by Granroth et al [6,

45]. 25

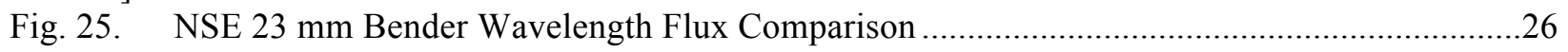

Fig. 26. NSE $60 \mathrm{~mm}$ Bender Wavelength Flux Comparison ...........................................................27

Fig. 27. NSE $120 \mathrm{~mm}$ Bender Wavelength Flux Comparison ........................................................28

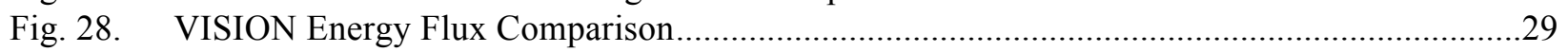

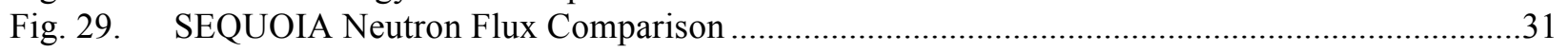

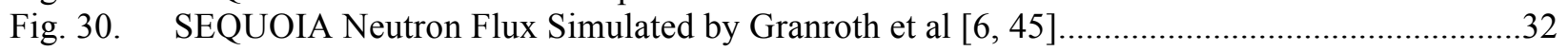

Fig. 31. Chopper Spectrometers Neutron Flux Measured by Stone [20] ............................................33

Fig. 32. Chopper Spectrometers Energy Resolution Measured by Stone [20].....................................3

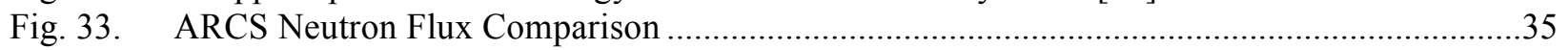

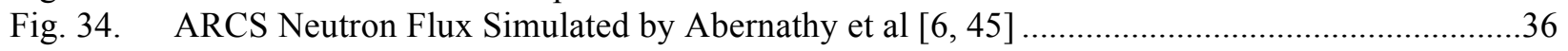

Fig. 35. Chopper Spectrometers Neutron Flux Measured by Stone [20] ...........................................37

Fig. 36. Chopper Spectrometers Energy Resolution Measured by Stone [20].....................................38 


\section{LIST OF TABLES}

Table

Page

Table 1: Summary of SAC Neutron Flux Recommendations.................................................................39 


\section{INTRODUCTION}

The Spallation Neutron Source (SNS) at Oak Ridge National Laboratory (ORNL) uses the Sample Activation Calculator (SAC) to calculate the activation of a sample after the sample has been exposed to the neutron beam in one of the SNS beamlines $[1,2]$. The SAC webpage takes user inputs (choice of beamline, the mass, composition and area of the sample, irradiation time, decay time, etc.) and calculates the activation for the sample. In recent years, the SAC has been incorporated into the user proposal and sample handling process, and instrument teams and users have noticed discrepancies in the predicted activation of their samples. The Neutronics Analysis Team validated SAC by performing measurements on select beamlines [3] and confirmed the discrepancies seen by the instrument teams and users. The conclusions outlined in [3] were that the discrepancies were a result of a combination of faulty neutron flux spectra for the instruments, improper inputs supplied by SAC (1.12), and a mishandling of cross section data in the Sample Activation Program for Easy Use (SAPEU) (1.1.2). This report focuses on the conclusion that the SAPEU (1.1.2) beamline neutron flux spectra have errors and are a significant contributor to the activation discrepancies. The results of the analysis of the SAPEU (1.1.2) flux spectra for all beamlines will be discussed in detail. The recommendations for the implementation of improved neutron flux spectra in SAPEU (1.1.3) are also discussed.

\section{SAC DESCRIPTION}

The SAC is used to calculate the activation of user samples after irradiation on any SNS beamline, and has been incorporated into the user proposal and sample handling process [2]. The SAC tool is a webpage that takes user inputs such as the choice of beamline, the mass, composition and area of the sample, irradiation time, decay time, etc. and invokes the SAPEU to calculate the activation of the sample. SAPEU uses the user input file (supplied by SAC) along with the CINDER 90 cross section library, a file containing the SNS beamline neutron flux spectra, and other reference files containing information on radiotoxicity, 2 X 2 sodium iodide detector efficiency, atomic mass, and natural isotopic abundances to calculate the activation of a given sample, and to provide handling guidance for the sample. The algorithm SAPEU uses to calculate the sample activation stems from the transmutation theory that is outlined in Lu's paper [2]. In order to effectively employ this algorithm, SAPEU makes several assumptions in order to decrease the calculation time of the sample activation. SAPEU assumes that there is a constant flux over time and over the sample volume, constant sample atom number, and no neutron reactions for the daughter of the direct activation productions [2]. SAC assumes irradiation for a single energy (wavelength) band at a single accelerator power level, for a large number of cycles between that power and zero power.

\section{ANALYSIS}

After analysis of the measurements taken on selected beamlines, we concluded that neutron flux spectrum used as an input to SAC was a significant contributor to the miscalculation of the sample activation [3]. The measured and simulated flux spectra for each beamline at the SNS were compared with the neutron flux spectrum used in SAC and recommendations for the change of the SAC neutron flux spectra are made based on this comparison. These recommendations are to be used on SAPEU (1.1.3).

\subsection{BEAMLINE 1A: USANS}

This beamline is currently not in SAC. 


\subsection{BEAMLINE 1B: NOMAD}

The NOMAD instrument is the Nanoscale-Ordered Materials Diffractometer at the SNS at ORNL [4]. Fig. 1 shows the comparison between a measured, simulated, and SAC input neutron flux spectra for the NOMAD instrument. Gallmeier's simulated spectrum was calculated with the Monte Carlo ray-tracing code, McStas, and used moderator source terms in [5,6]. A comparison between Gallmeier's simulated spectrum and Neuefeind's simulated neutron flux spectrum (calculated with IDEAS [4]) is shown in Fig. 2 and gives validation to Gallmeier's simulation [5,6]. Fig. 3 shows Neuefeind's IDEAS simulated neutron current spectrum compared with a separate measurement taken when SNS was running with an average beam power of $896 \mathrm{~kW}$ (not Iverson's measurement shown in Fig. 1) [4]. The agreement in Fig. 3 provides further validation of the simulated spectra. Iverson's measured neutron flux spectrum used in Fig. 1 has uncertainties about the area that was used during the measurement.

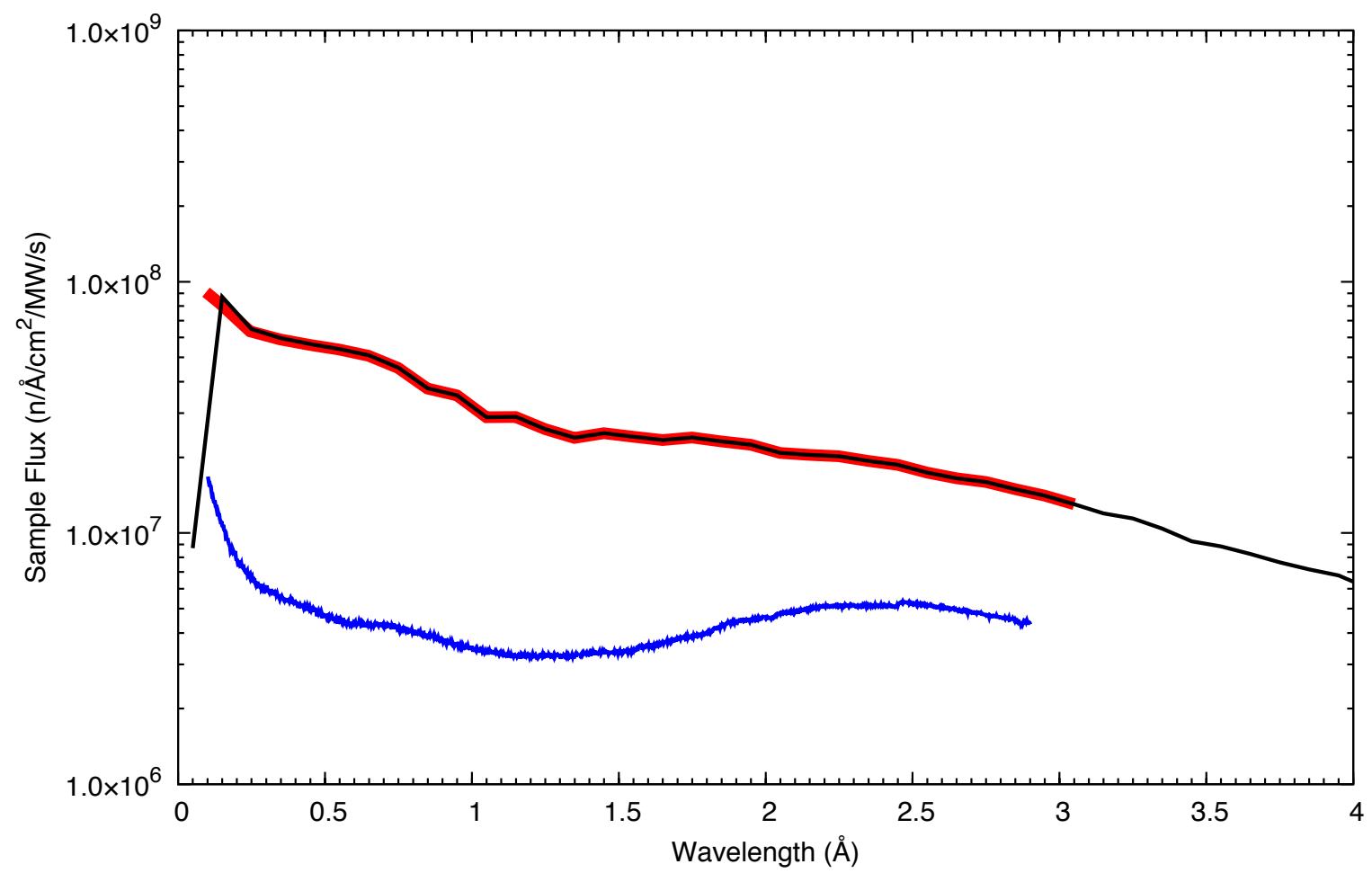

SAC

2011-10-17 Iverson Measured 2010-09-09 Gallmeier Simulated

Fig. 1. NOMAD Neutron Flux Comparison 


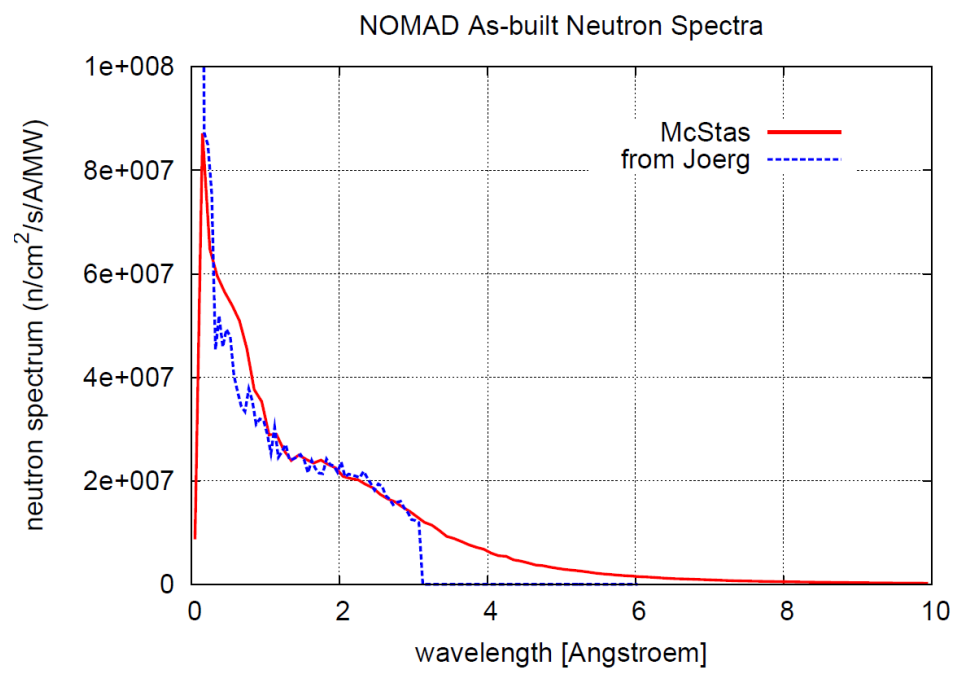

Fig. 2. Comparison of Simulated NOMAD Neutron Fluxes by Mcstas/Gallmeier $[5,6]$ and IDEAS/Neuefeind (from Joerg) [4]

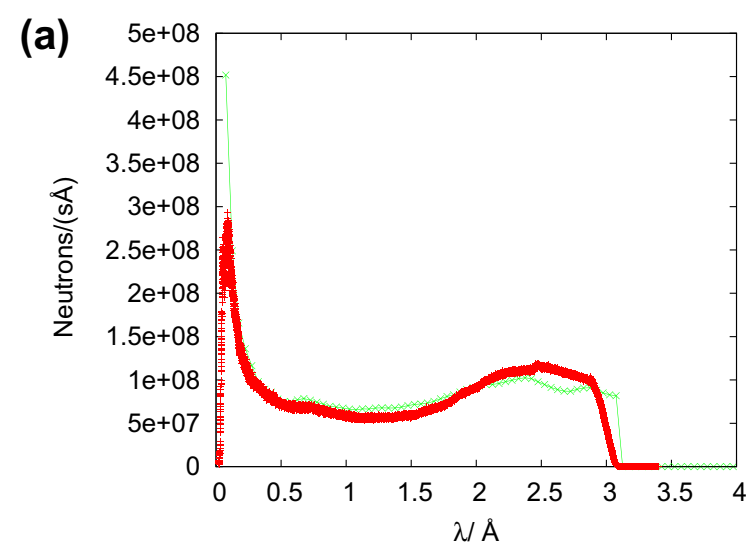

Fig. 3. Comparison of measured (red) and simulated (green) NOMAD neutron current by Neuefeind et al [4]

Considering the uncertainty about the measured spectra, the Neutronics Analysis Team implements no change in the neutron flux spectrum for the NOMAD instrument that is currently in SAC for SAPEU (1.1.3) and documents that the neutron flux spectrum that is currently in SAC is Gallmeier's simulated neutron flux spectrum $[5,6]$. The large differences between measured and simulated spectra could also hint on some guide misalignment. In this case discrepancies between measured and simulated sample activities can be attributed to the spectra mismatch.

\subsection{BEAMLINE 2: BASIS}

The BASIS instrument is the Backscattering Silicon Spectrometer at the SNS at ORNL [7]. Fig. 4 shows the comparison between the measured neutron flux spectrum by Mamontov [7] and the SAC Version 1.11 neutron flux spectrum for the BASIS instrument. The origins of the SAC spectrum are 
unknown. The measured flux spectrum given in arbitrary units (shown in Fig. 5) was normalized to give an integrated flux value of $1.3 \mathrm{E} 07 \mathrm{n} / \mathrm{cm}^{2} / \mathrm{s}$ over a wavelength band of $0.5 \AA$, centered at $6.4 \AA$ with a 60 $\mathrm{Hz}$ repetition rate [7]. The measured neutron flux spectrum shown in Fig. 4 matches closely with the current input neutron flux spectrum for SAC, and the measured spectrum provides data with a larger range and greater detail.

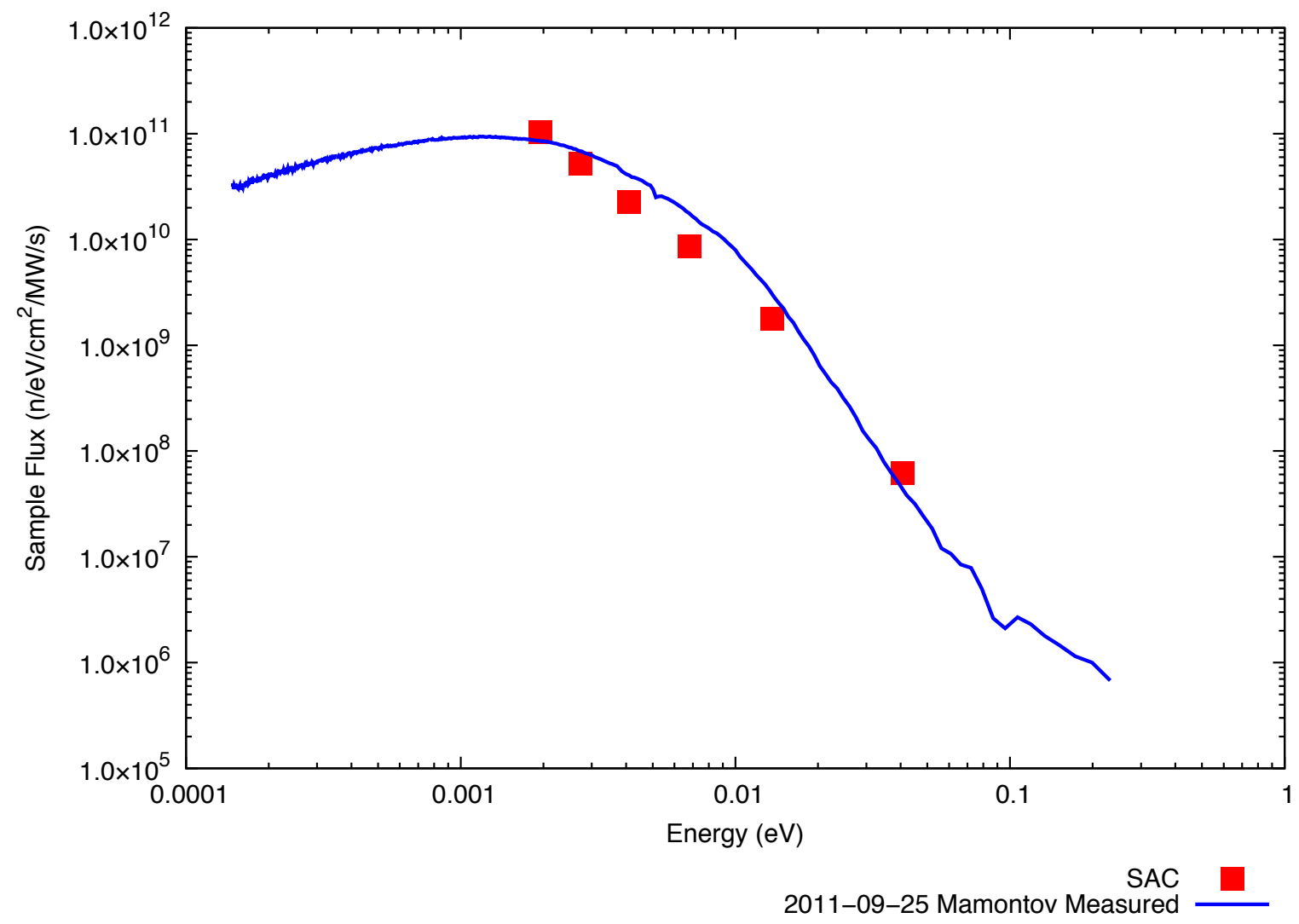

Fig. 4. BASIS Neutron Flux Comparison 


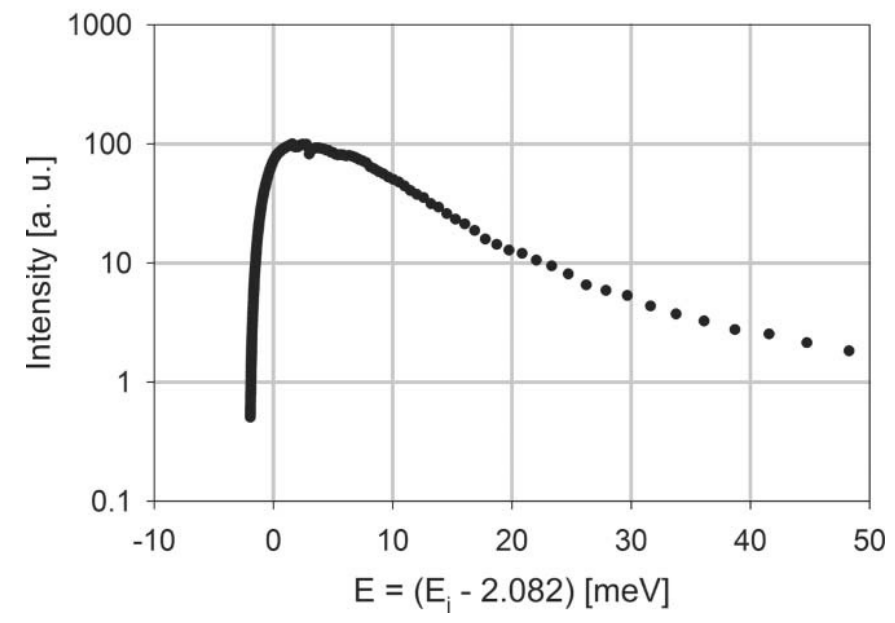

Fig. 5. BASIS Neutron Flux as Measured by Mamontov [7].

Upon the results of the neutron flux spectrum comparison, the Neutronics Analysis Team implements the measured neutron flux spectrum shown in Fig. 4 in SAPEU (1.1.3) for the BASIS instrument [7].

\subsection{BEAMLINE 3: SNAP}

The SNAP instrument is the Spallation Neutrons and Pressure Diffractometer at the SNS at ORNL [8]. Fig. 6 shows the comparison between a measured neutron flux spectrum and the neutron flux spectrum used as an input to SAC for the SNAP instrument. The measured neutron flux spectrum was measured with a well-characterized beam monitor at the sample position with a beam area of $11.4 \mathrm{~cm}^{2}$ and an average power of $0.59 \mathrm{MW}$ on November 7, 2008 [9]. The measured neutron flux spectrum shown in Fig. 6 matches closely with the current input neutron flux spectrum for SAC, and the measured spectrum provides data with greater detail. 


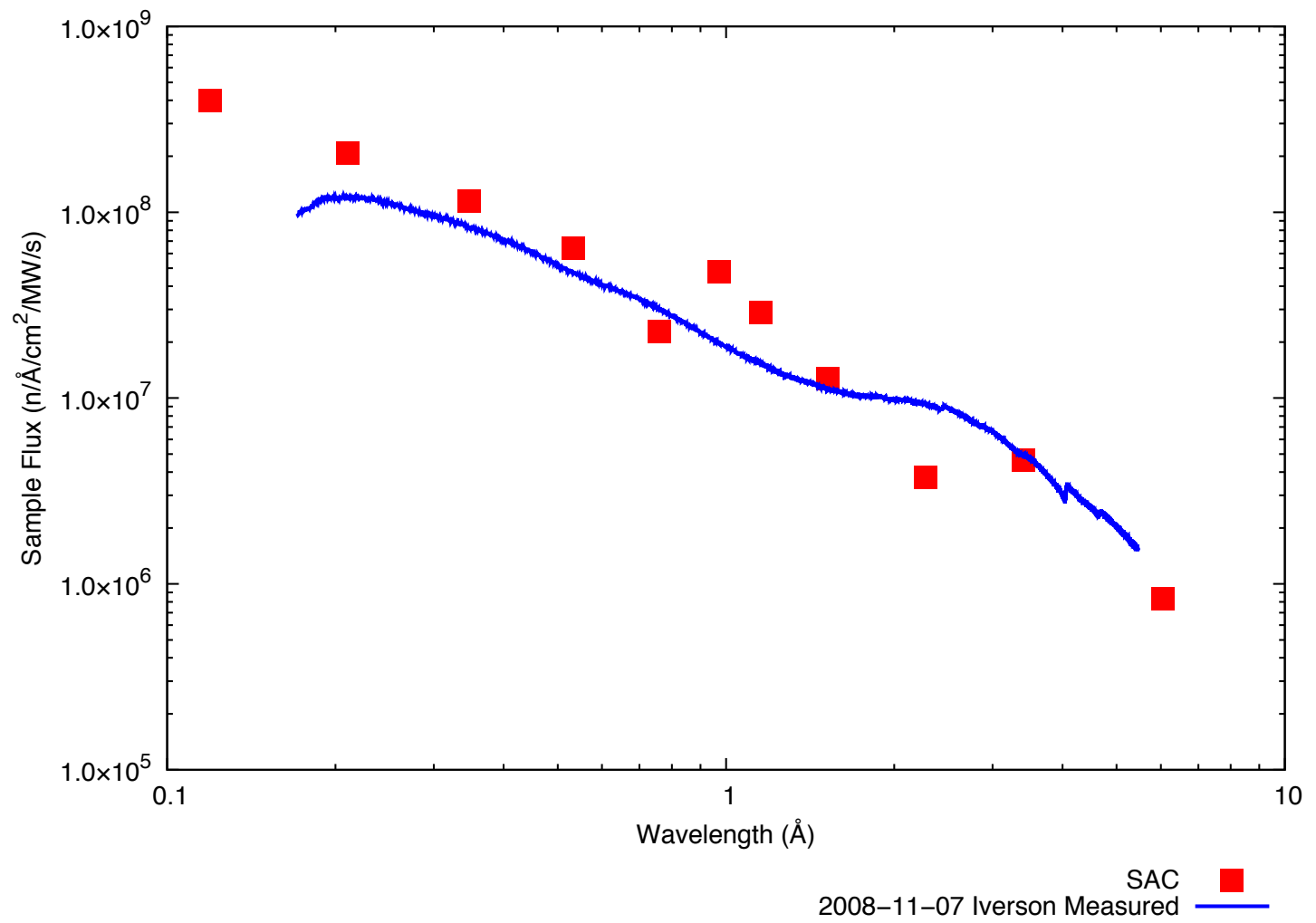

Fig. 6. SNAP Wavelength Flux Comparison

Upon the results of the neutron flux spectrum comparison in Fig. 6, the Neutronics Analysis Team implements no change to the neutron flux spectrum in SAPEU (1.1.3) for the SNAP instrument because of the validation by the measured neutron flux spectrum shown in Fig. 6, and documents that the neutron flux in SAPEU (1.1.2) and (1.1.3) is P.D. Ferguson's simulation [10].

\subsection{BEAMLINE 4A: MR}

The MR instrument is the Magnetic Reflectometer at the SNS at ORNL [11]. Fig. 7 shows the comparison between a measured neutron flux spectrum and the neutron flux spectrum used as an input to SAC for the MR instrument [12]. The measured neutron flux spectrum used in Fig. 7 is a measurement of an unpolarized neutron beam with $30 \mathrm{~mm}$ vertical slits and $3 \mathrm{~mm}$ horizontal slits and was measured with a beam monitor located at the sample position, and is a result of the detector TOF counts that are normalized by power and detector area in order to arrive at flux [13]. While a polarized beam is not shown in Fig. 7, Lauter states that the polarization of the beam implements a linear $50 \%$ reduction in the intensity of the neutron flux spectrum [14]. In order to have a conservative estimate of the neutron flux intensity, Lauter typically does not include the factor of 2 reduction in neutron flux intensity given by the collimation of the neutron beam in her calculations of sample activation [14]. The measured neutron flux spectrum shown in Fig. 7 differs by a factor of 50 with the SAC input neutron flux spectrum. The SAC spectral shape was consistent with the theoretical spectral shape of the neutron flux distribution coming 
from a supercritical hydrogen moderator. The beamline scientist for the MR instrument speculates that the SAC neutron flux distribution was likely calculated with early developmental Monte Carlo models. The SAC neutron flux has been scaled to the magnitude of the measurement at $5 \AA$ with a scaling factor of 2.131E-02 applied to the SAC neutron flux spectrum and the results are shown in Fig. 7.

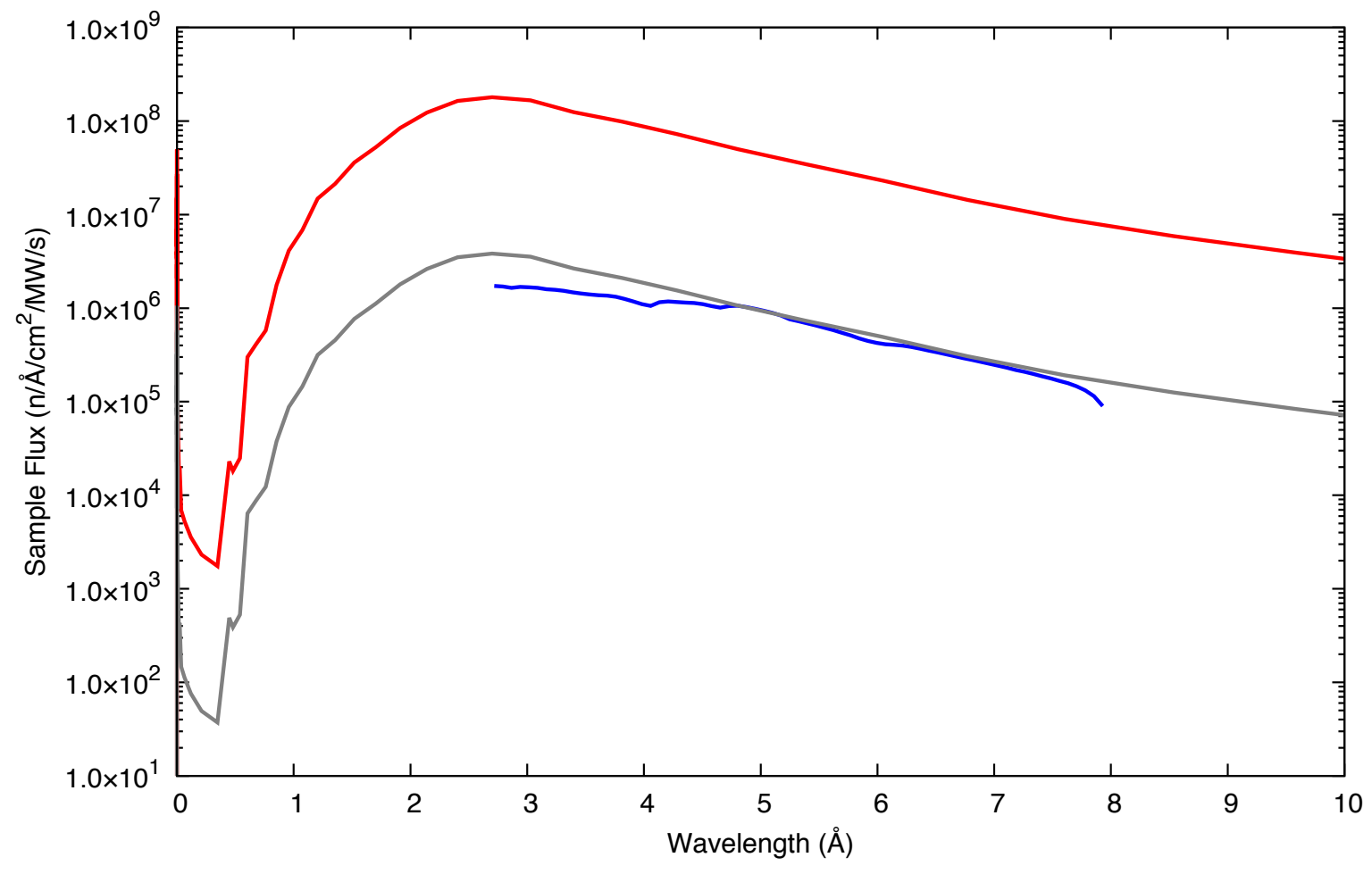

2014-06-19 Lauter Measured

Scaled SAC Flux

Fig. 7. MR Neutron Flux Wavelength Comparison

Upon the results of the neutron flux spectrum comparison, the Neutronics Analysis Team implements the scaled SAC flux spectrum shown in Fig. 7 in SAPEU (1.1.3) for the MR instrument [14].

\subsection{BEAMLINE 4B: LR}

The LR instrument Liquids Reflectometer at the SNS at ORNL [15]. The comparison of the measured and SAC neutron flux spectra is shown in Fig. 8 for the LR instrument [16]. The measured neutron flux spectrum shown in Fig. 8 was measured with a well-characterized detector at the sample position with the beam extent slits (S2) set to $34.8 \mathrm{~mm}$ for the horizontal slit and $5.0 \mathrm{~mm}$ for the vertical slit, and the divergence slits set to $35.0 \mathrm{~mm}$ for the horizontal slit and $10.0 \mathrm{~mm}$ for the vertical slit [16]. The measurement was taken with the bandwidth choppers set to allow a wavelength band of 2.5 to $5.5 \AA$ to pass to the sample position [16]. The SAC input neutron flux spectrum was scaled to the measured 
neutron flux at $4 \AA$ based on the same logic as the scaling of the neutron flux for the MR instrument. The scaling factor applied to the SAC neutron flux spectrum is 7.810E-02. The SAC input neutron flux spectrum has also been speculated to originate from early developmental Monte Carlo models.

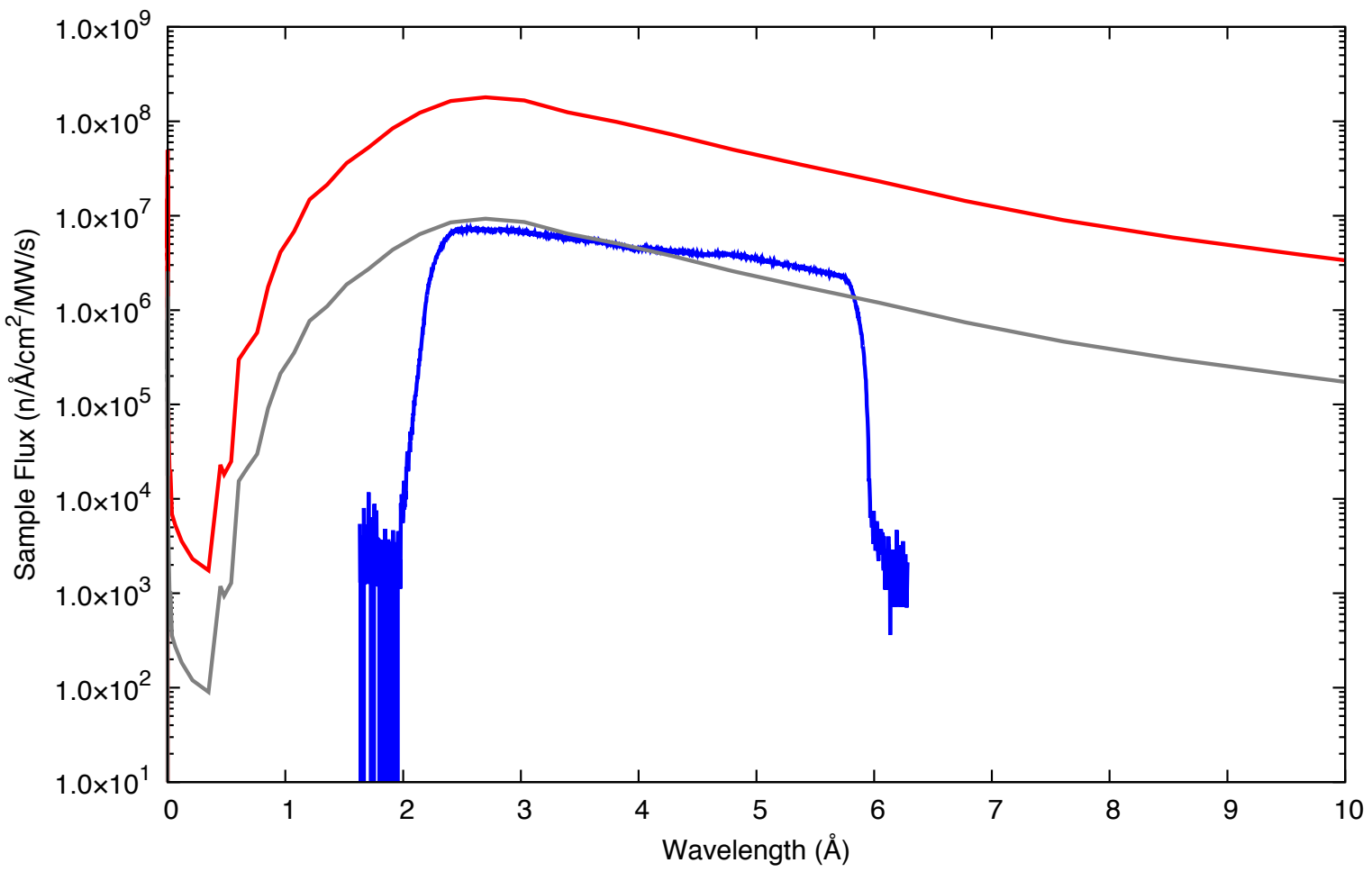

2008-09-10 Iverson Measured Scaled SAC Flux

Fig. 8. LR Wavelength Flux Comparison

Upon the results of the neutron flux spectrum comparison, the Neutronics Analysis Team implements the scaled SAC flux spectrum shown in Fig. 8 in SAPEU (1.1.3) for the LR instrument [16].

\subsection{BEAMLINE 5: CNCS}

Fig. 9 shows the comparison between several neutron flux spectra for the Cold Neutron Chopper Spectrometer (CNCS) instrument at SNS [17]. All four of the neutron flux spectra shown in Fig. 9 have been measured or simulated using the high flux setting and normalized by the full instrument energy resolution at the high flux setting shown in Fig. 10 [17]. The full instrument energy resolution was measured in both the high flux and high resolution settings with the Fermi chopper running at $180 \mathrm{~Hz}$ and the sample chopper running at $300 \mathrm{~Hz}$ [17]. The high flux and high resolution settings differ only in the selection of the chopper slits that are paired [17]. The SAC neutron flux spectrum is currently used by $\mathrm{SAC}$ to calculate the expected activation of a sample on CNCS. Iverson's measurement of flux at the 
sample position was done with a calibrated beam monitor at the sample position $[18,19]$. Ehlers' simulated neutron flux spectrum shown in Fig. 9 is the dashed line shown in Fig. 11 [17]. The dashed line in Fig. 11 is a result of a function that has been fitted to Iverson's measurement and the McStas stimulation shown in Fig. 11 in order to best approximate the flux for the CNCS instrument [17]. Stone's measured spectrum is the integrated beam monitor intensity (measured immediately before the sample position) multiplied by the conversion from Coulombs to neutrons and divided by the average power, beam area, and the corresponding energy dependent energy resolution [20]. The discontinuities in Stone's spectrum are a result of aluminum's Bragg edges around 4 and $20 \mathrm{meV}$. The differences in magnitude between Iverson's measurement and Stone's measurements can be attributed to the installation of a new guide segment on the CNCS instrument $[18,20]$

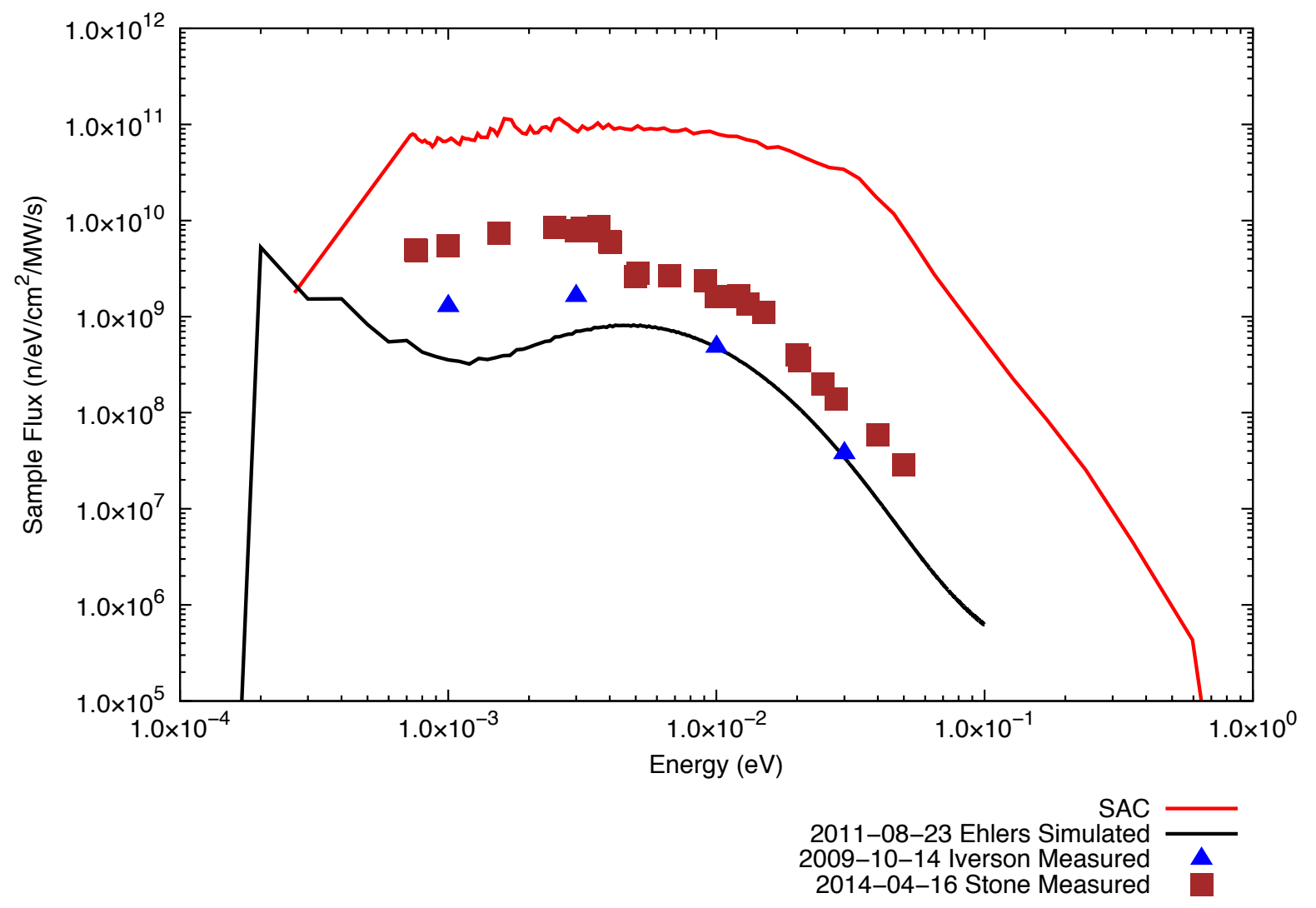

Fig. 9. CNCS Neutron Flux Spectra Comparison 


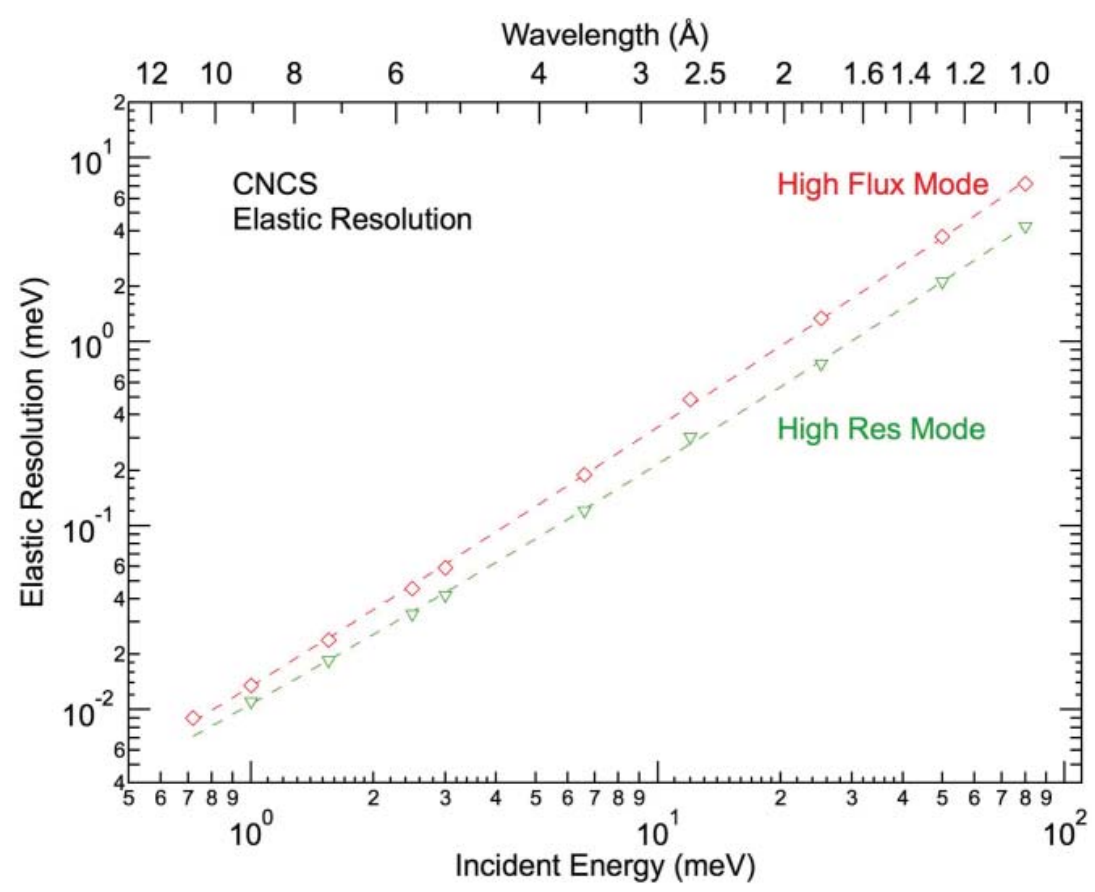

Fig. 10. CNCS Energy Resolution from Ehler [17]

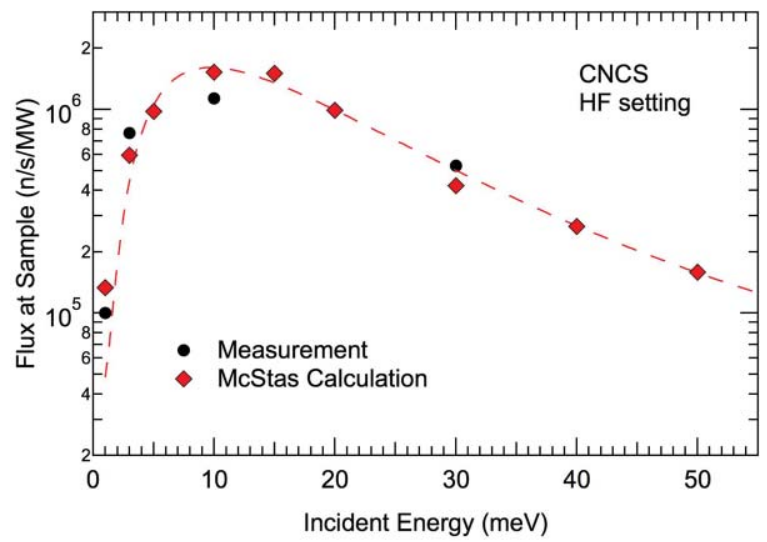

Fig. 11. CNCS Neutron Flux from Ehler [17]

Due to the comparison made in Fig. 9, The Neutron Analysis Team implements Stone's measured neutron flux spectrum in SAPEU (1.1.3) for the CNCS instrument [20]. 


\subsection{BEAMLINE 6: EQ-SANS}

The EQ-SANS instrument is the Extended Q-Range Small-Angle Neutron Scattering Diffractometer at SNS [21]. The neutron flux spectrum used in the SAC tool is compared with Gallmeier's simulated spectra for $100 \%$ para $\mathrm{H}$ and $30 \%$ ortho and $70 \%$ para $\mathrm{H}$ and Iverson's measurement in Fig. 12 [6, 22, 23]. Iverson's measured neutron flux shown in Fig. 12 is a combination of measurements taken over several wavelength bands and the corresponding chopper cutoffs have been removed. These measurements were done using a calibrated beam monitor located just upstream of the nominal sample position while SNS was operating at $1 \mathrm{MW}$ and the Sample Pinhole set to $10 \mathrm{~mm}$ diameter and the Source Pinhole set to $25 \mathrm{~mm}$ radius [23]. It should be noted that Iverson's measured neutron flux was normalized by the area of the Sample Pinhole rather than the area of the beamspot at sample position [23].

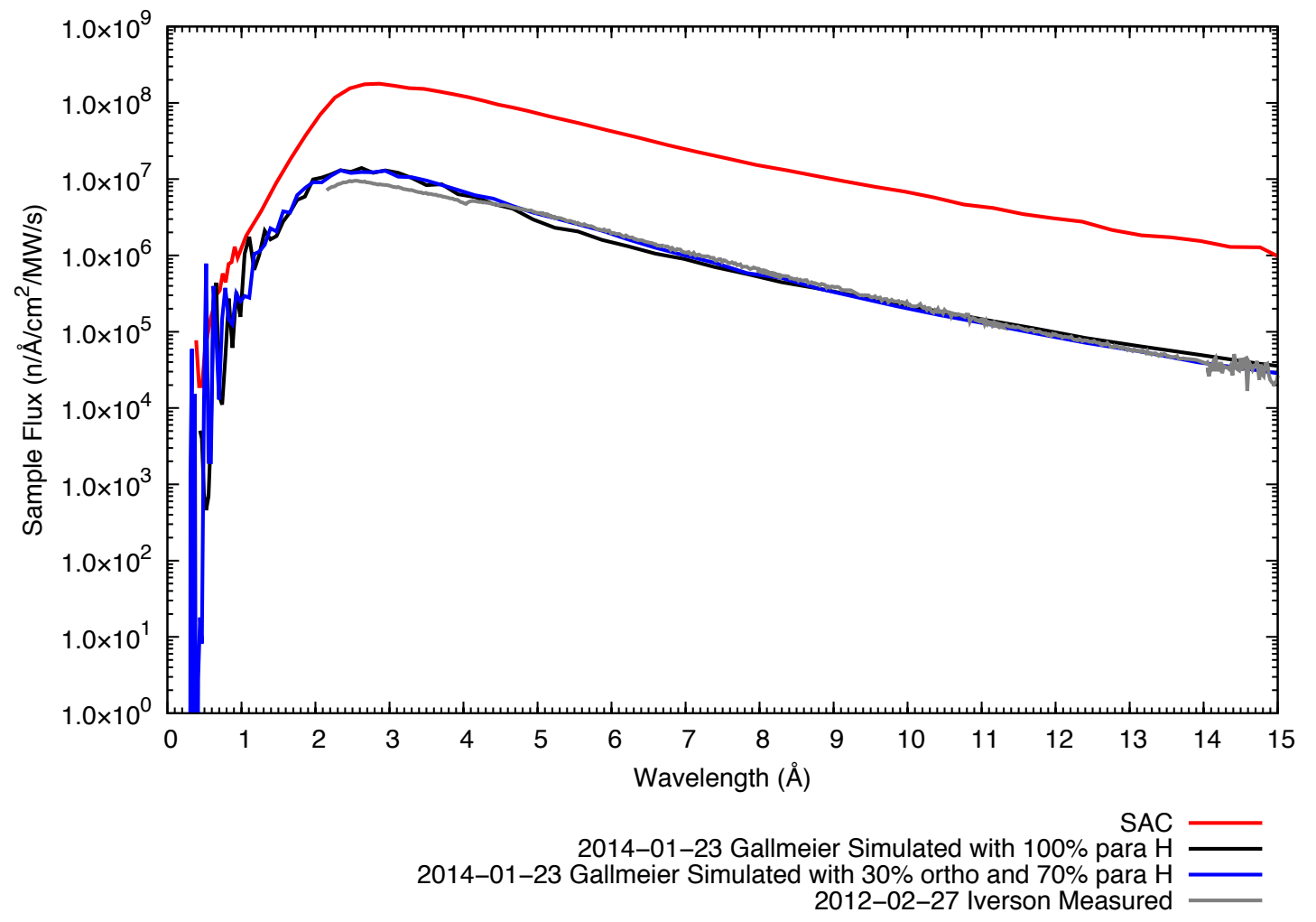

Fig. 12. EQ-SANS Wavelength Flux Comparison *

The Neutronics Analysis Team implements Gallmeier's $100 \%$ para hydrogen simulation in SAPEU (1.1.3) for the EQ-SANS instrument [6, 22].

\footnotetext{
${ }^{*}$ The chopper cutoffs have been removed from the measured neutron flux spectrum.
} 


\subsection{BEAMLINE 7: VULCAN}

The VULCAN instrument is the Engineering Materials Diffractometer at the SNS [24]. The neutron flux spectrum used in SAC is compared with a Gallmeier's simulated neutron flux spectrum and Iverson's measured neutron flux spectrum for the VULCAN instrument in Fig. 13 [5, 6, 25]. The simulated and measured spectra in Fig. 13 nominally differ with the SAC input spectrum by approximately a factor of two. Iverson's measured neutron flux spectrum was measured with a calibrated beam monitor located at the nominal sample position viewing a $2.32 \mathrm{~mm}$ diameter pinhole with the default chopper settings and the upstream guide configuration in the High-Intensity mode [25].

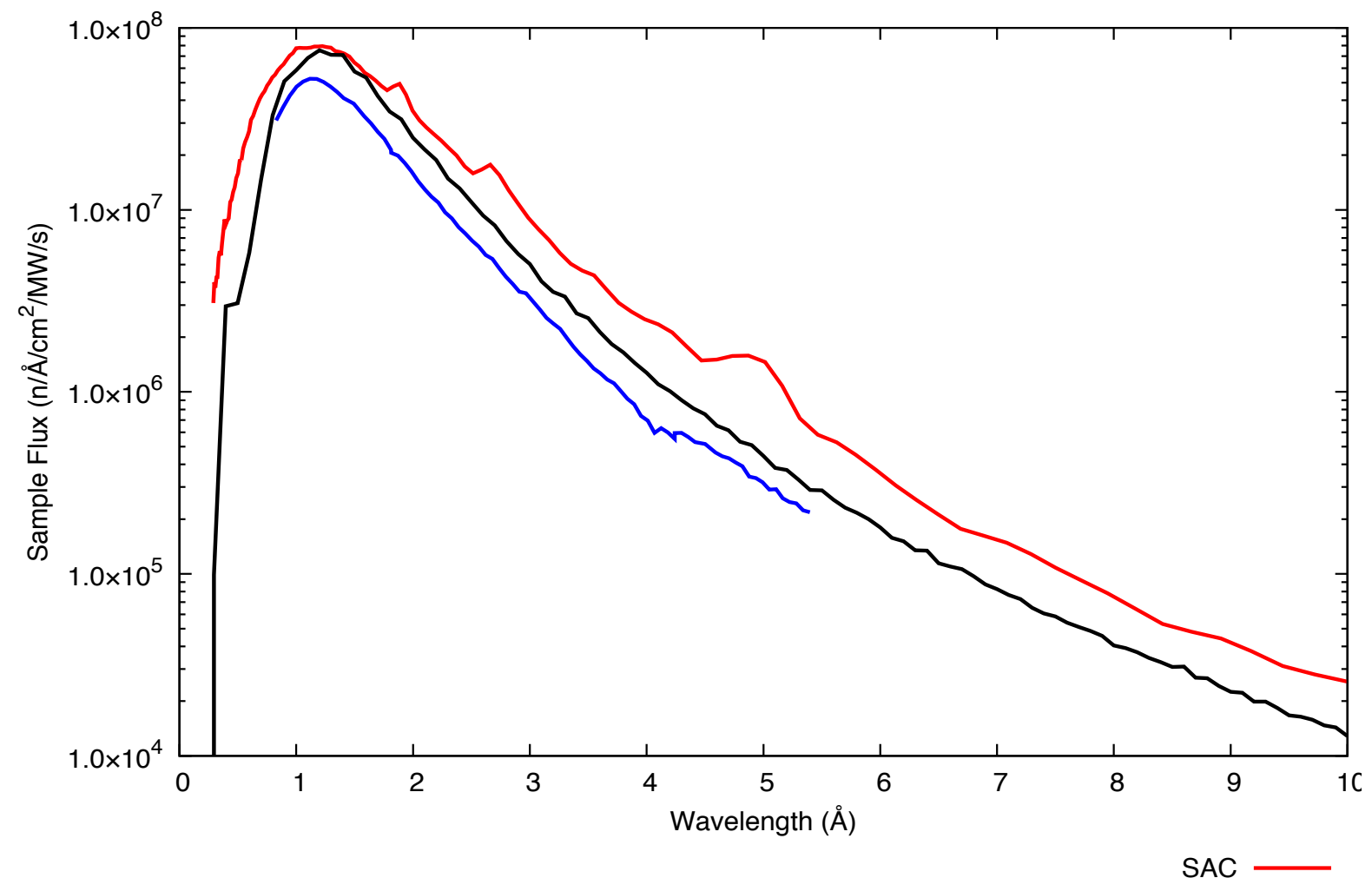

2013-10-16 Iverson Measured 2014-05-01 Gallmeier Simulated

Fig. 13. VULCAN Wavelength Flux Comparison ${ }^{\dagger}$

Based on the comparisons made above in Fig. 13, the Neutronics Analysis Team implements Gallmeier's simulated spectrum in SAPEU (1.1.3) for the VULCAN instrument [5,6].

\footnotetext{
${ }^{\dagger}$ The chopper cutoffs have been removed from the measured neutron flux spectrum.
} 


\subsection{BEAMLINE 8A}

This beamline is currently not in SAC

\subsection{BEAMLINE 8B}

This beamline is currently not in SAC

\subsection{BEAMLINE 9: CORELLI}

The CORELLI instrument is the elastic diffuse scattering spectrometer at the SNS [26]. Fig. 14 shows the comparison between the Ye's simulated neutron flux spectrum and Iverson's measured neutron flux spectrum for the CORELLI instrument [27]. Ye uses a McStas model to calculate the spectrum shown below at the nominal sample position [27]. Iverson's measured neutron flux was measured with a calibrated beam monitor located at the nominal sample position [27]. Reports of both the measured and simulated neutron flux spectra are currently being written.

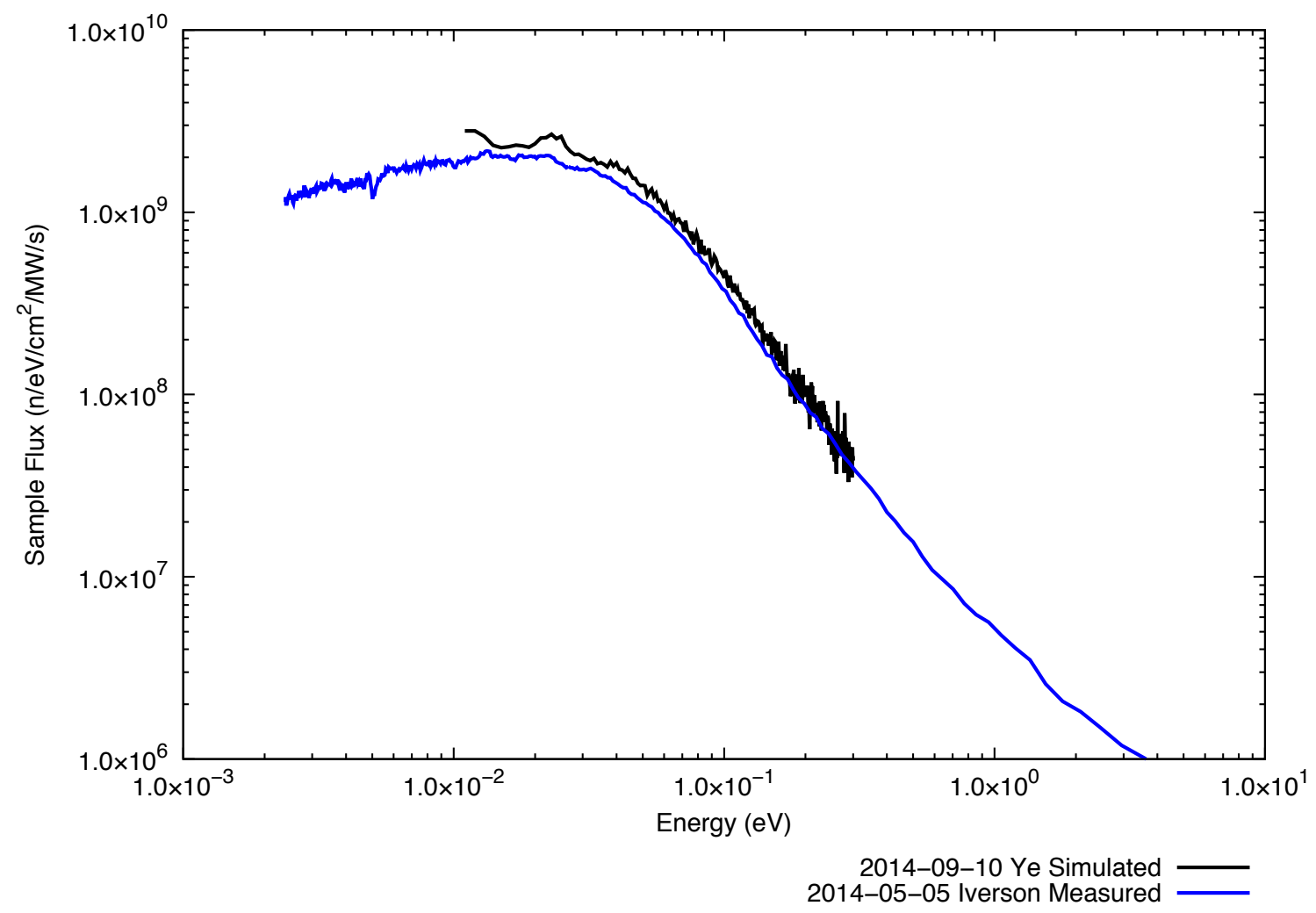

Fig. 14. CORELLI Energy Flux Comparison 
Based on the comparison in Fig. 14 and the two spectra's agreement, the Neutronics Analysis Team implements Iverson's measured neutron flux spectrum in SAPEU (1.1.3) for the spectrum's extended energy range [27].

\subsection{BEAMLINE 10}

This beamline is currently not in SAC

\subsection{BEAMLINE 11A: POWGEN}

The POWGEN instrument is the third-generation high-resolution high-throughput powder diffraction instrument at the SNS [28]. Fig. 15 and Fig. 16 show the comparisons between the SAC, simulated, and measured neutron flux spectra for the POWGEN instrument in the high intensity and high resolution settings [28], respectively. The SAC neutron flux spectrum is currently used by SAC to calculate the expected activation of a sample after being irradiated on POWGEN. The origins of this spectrum are not documented. Gallmeier's simulated neutron flux spectrum, shown in Fig. 15, was simulated using an asbuilt McStas model developed by Hamilton and Gallmeier [5, 6]. Iverson's measurements in Fig. 15 and Fig. 16 were measured with a calibrated beam monitor and the default chopper settings at the nominal sample position [29]. Hodges' simulated flux spectrum was simulated using a McStas model developed by Hodges [30]. Iverson reports that a $5 \%$ gain in intensity of the high intensity setting measurements could be achieved by the fine-tuning of the position of the guide translation table position [29]. However, the $5 \%$ increase in the high intensity setting measurements still leaves a significant difference between the measured and simulated neutron flux spectra. Gallmeier notes differences in the measured and simulated neutron flux spectra in Fig. 15 and Fig. 16 could be attributed to misalignments of the guide when it was installed $[5,6]$. The measured and simulated neutron flux spectra shown in Fig. 15 and Fig. 16 have been normalized to average beam power, beam area, and energy bin width. 


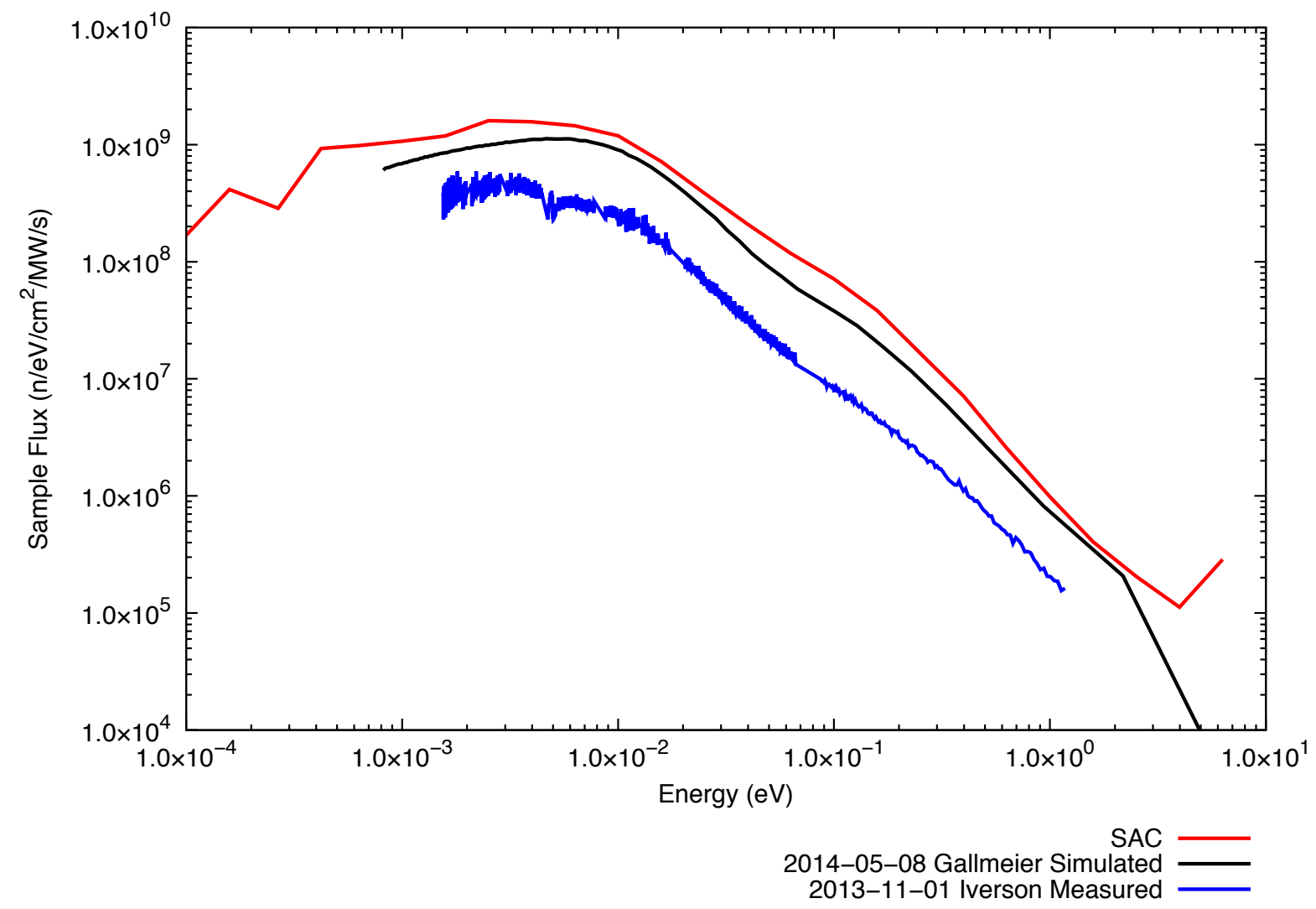

Fig. 15. POWGEN High Intensity Energy Flux Comparison 


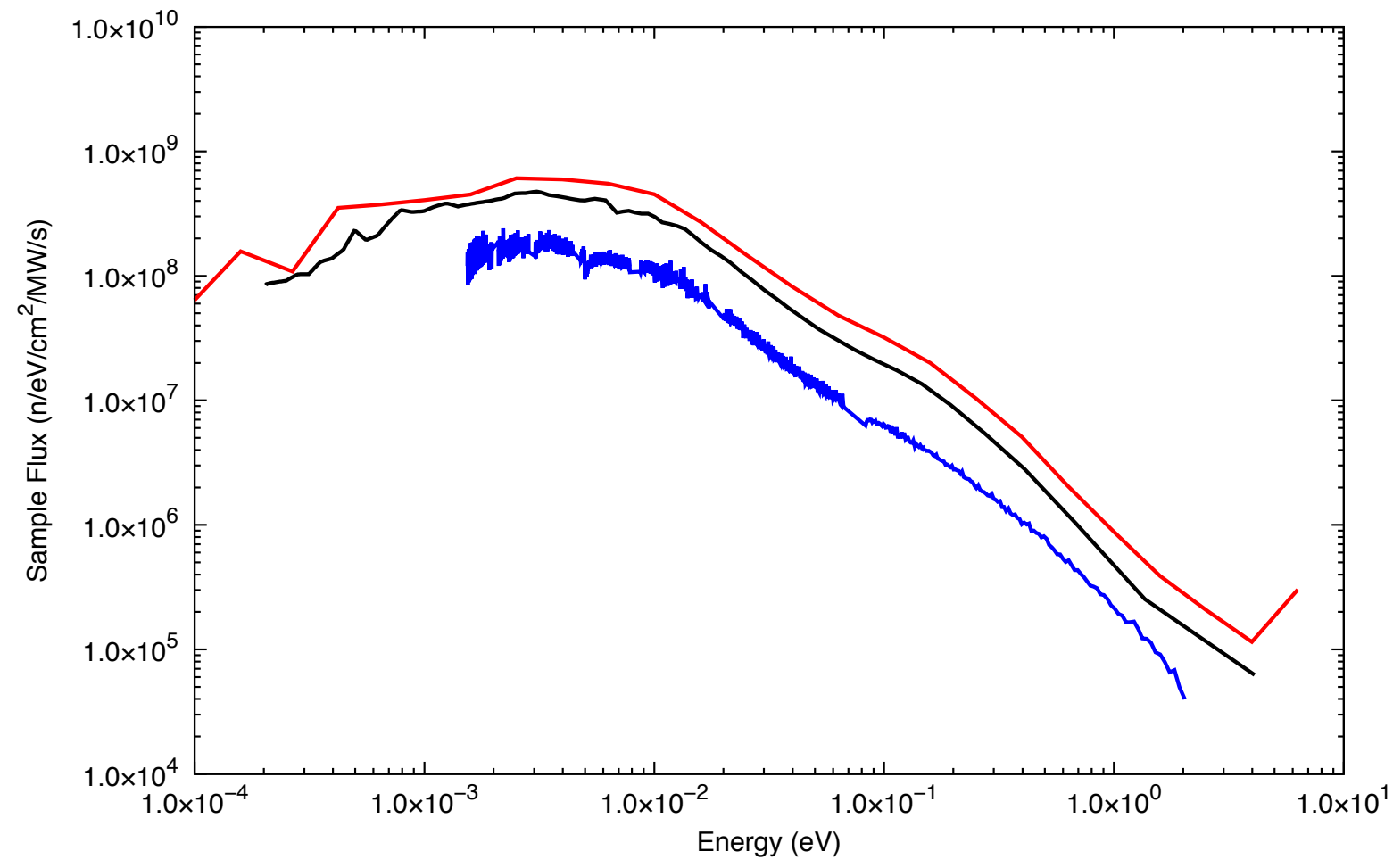

2009-10-01 Hodges Simulated 2013-11-01 Iverson Measured

Fig. 16. POWGEN High Resolution Energy Flux Comparison

Based on the comparisons shown in Fig. 15 and Fig. 16, the Neutronics Analysis Team implements Gallmeier's simulated neutron flux spectrum for POWGEN in the High Intensity setting in SAPEU (1.1.3) and Hodges' simulated neutron flux spectrum for POWGEN in the High Resolution in SAPEU (1.1.3) pending a gold foil activation measurements on the POWGEN instrument $[5,6,30]$.

\subsection{BEAMLINE 11B: MANDI}

MaNDi is the macromolecular neutron diffractometer at the SNS [31]. Fig. 17 shows the comparison of several neutron flux spectra measured and simulated at both the sample position and the guide exit. The Frost measured neutron flux spectrum was measured with a beam monitor at the sample position with a 3 $\mathrm{mm}$ diameter aperture with the instrument operating in a $30 \mathrm{~Hz}$ mode [32]. The factor of 2 in intensity of the spectrum as a result of the $30 \mathrm{~Hz}$ operation of the instrument has been accounted for in the normalization. Gallmeier's simulated neutron flux spectrum at the sample position and the guide exit are computed from the same McStas model, and have been normalized by average beam power, beam area, and bin size [33]. Iverson's measurement was taken at the guide exit with a well-calibrated beam monitor 
with the instrument running at a $5 \mathrm{~Hz}$ mode with all of the choppers parked in the nominally open position [34]. Coates' simulated neutron flux spectrum is computed from a McStas model at the guide exit and has been normalized to $1 \mathrm{MW}$ power [35]. Both Iverson and Coates' spectra have been normalized to the area of the beam at the guide exit of $0.49 \mathrm{~cm}^{2}$ [33]. The Gallmeier and Coates' simulated neutron flux spectra at the guide exit are in good agreement, which sets the basis for the comparison of Coates' simulated flux and Gallmeier's simulated flux at the sample position. Coates' simulated neutron flux spectrum at the guide exit and Gallmeier's simulated neutron flux spectrum at the sample position are in good agreement at higher energies but the difference in the two spectra increases with decreasing energy. This is because the divergence of the neutrons increase with decreasing energy [33]. Frost's measurement and Gallmeier's simulation at the sample position are also in good agreement.

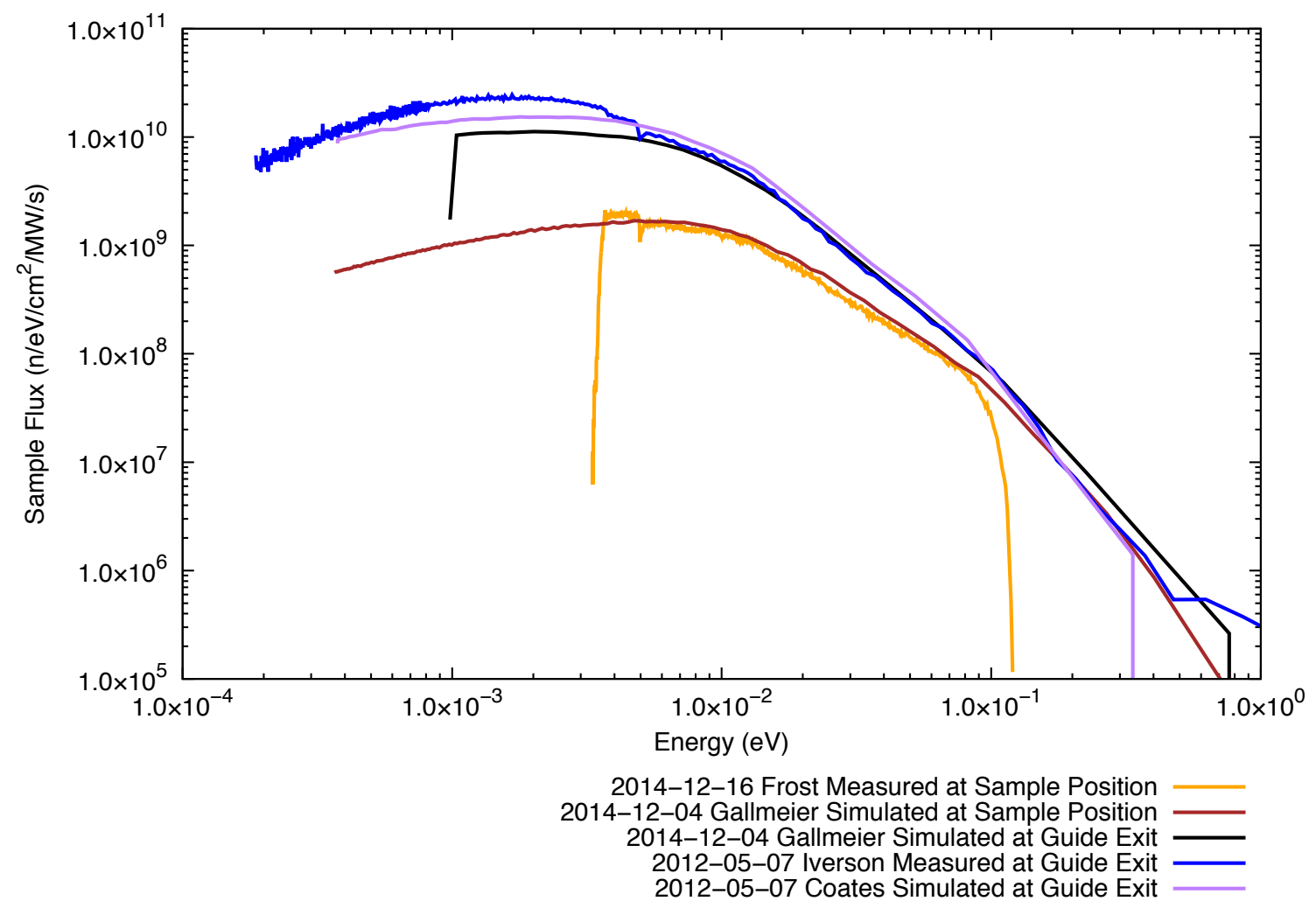

Fig. 17. MANDI High Intensity Energy Flux Comparison

Based on the comparisons made in Fig. 17, the Neutronics Analysis Team implements Gallmeier's simulated spectrum at the sample position in SAPEU (1.1.3) for the MaNDi instrument [33]. 


\subsection{BEAMLINE 12: TOPAZ}

TOPAZ is the single-crystal neutron diffractometer at the SNS [36]. Fig. 18 shows the flux comparison between the SAC neutron flux spectrum and several measured and simulated spectra. It should be noted that the origins of the SAC neutron flux spectrum are unknown. The Wang simulated spectrum shown in Fig. 18 was simulated with a McStas model, modified to the SNS 2014A run cycle neutron guide and slits configuration $[6,37]$. Wang's spectrum was calculated at the sample position with a beam monitor, the simulation did not include bandwidth choppers, and the spectrum has been normalized to a $2.54 \mathrm{~mm}$ diameter BN aperture [6,37]. The Iverson measured spectrum in Fig. 18 is a result of a measurement of the wavelength range with $1.8 \AA$ as the center wavelength using a wellcharacterized beam monitor located at the sample position, and the spectrum has been normalized to the average power during the measurement and the beam area at the sample position [38]. Iverson's measurement was taken after the guide realignment documented in the referenced paper in Section III.B $[5,6]$. The agreement between Wang and Gallmeier's spectra provides further validation of Wang's simulated neutron flux spectrum. Frost's measurement shown in Fig. 18 was measured with a beam monitor located at the sample position [32]. The measured spectrum has been normalized to a beam aperture of $2.4 \mathrm{~mm}$ diameter [32]. The Frost and Iverson measurement are very similar. At higher energies, both measured spectra deviate more and more from the simulations. This is because the simulations assume a "perfect" guide mirror surface, whereas the measurements show the reality that the neutron guide mirrors are not "perfect," but "wavy." The imperfections in the neutron guide system are amplified at the higher neutron energies, thus the increase in the discrepancies between the measurements and simulations. 


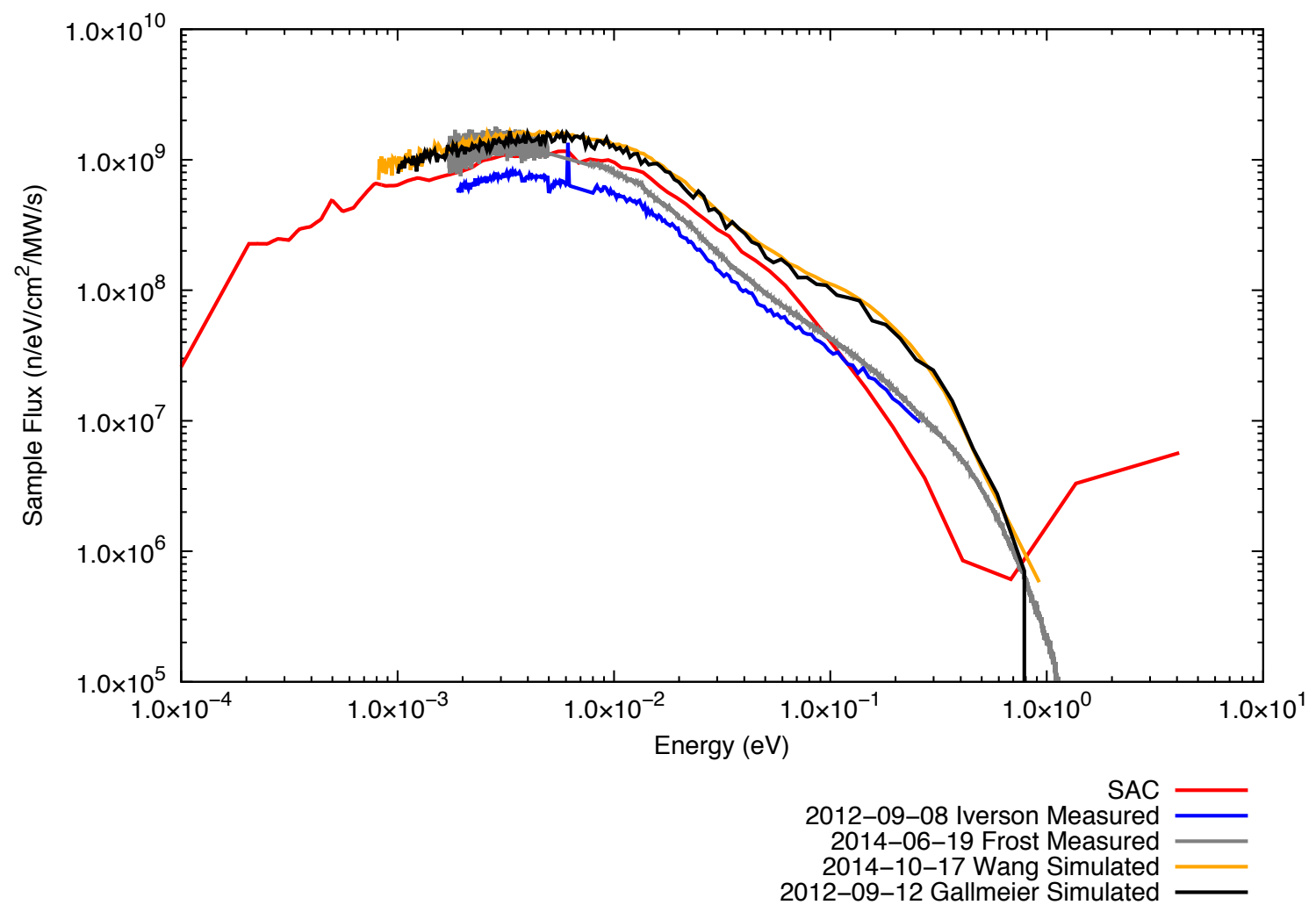

Fig. 18. TOPAZ High Intensity Energy Flux Comparison

Based on the comparisons shown in Fig. 18, the Neutronics Analysis Team implements Wang's simulated spectrum in SAPEU (1.1.3) for the TOPAZ instrument [37]. By using Wang's simulated neutron flux spectrum, it is assured that there will be slight over-prediction of the sample activation.

\subsection{BEAMLINE 13: FNPB}

The comparison of the Fundamental Neutron Physics Beamline (FNPB) neutron flux vs. wavelength is shown in Fig. 19 [39]. The origins of the SAC input neutron flux spectrum are unknown but speculated to come from a McStas simulation documented in [40]. Iverson's measurement, shown in Fig. 19, was measured with a well characterized transmission monitor that was masked with borated aluminum to view a $5 \mathrm{~mm}$ diameter pinhole, and was mounted $0.15 \mathrm{~m}$ downstream of the guide exit [41]. 


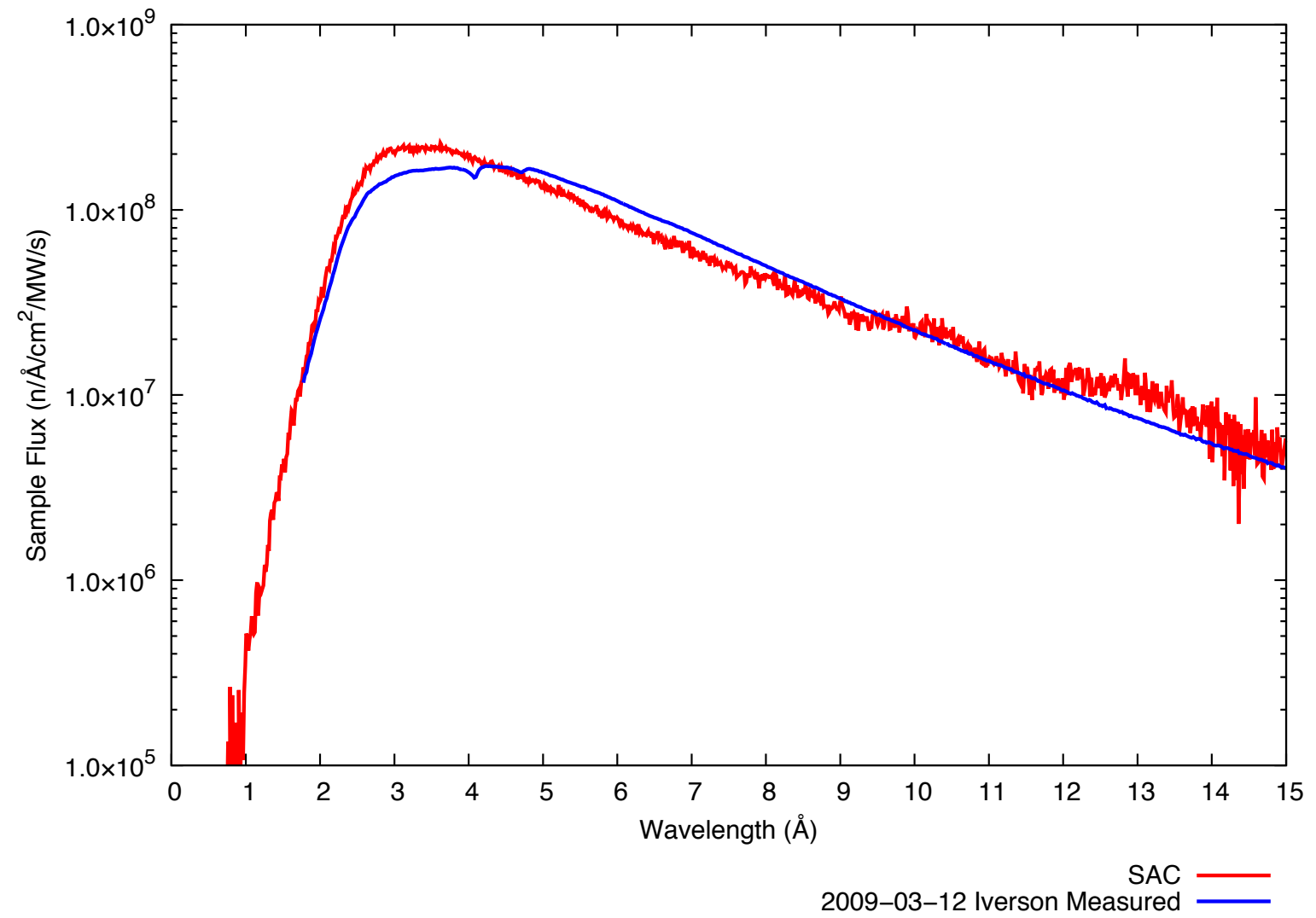

Fig. 19. FNPB Neutron Flux Spectra Comparison at $15 \mathrm{~cm}$ Distance From the Neutron Guide Exit.

Based on the comparisons shown in Fig. 19, the Neutronics Analysis implements Iverson's measurement (shown in Fig. 19) in SAPEU (1.1.3) for the FNPB instrument because of the spectrum's documentation and validation [41].

\subsection{BEAMLINE 14A}

This beamline is currently not in SAC

\subsection{BEAMLINE 14B: HYSPEC}

The HYSPEC instrument is the crystal time-of-flight hybrid spectrometer at the SNS with polarization capabilities [42]. Fig. 20 shows the comparison between the SAC input flux spectrum and several measured spectra for the HYSPEC instrument. The HYSPEC instrument is a hybrid spectrometer because it blends a high-intensity, medium-resolution, cold to thermal direct geometry chopper spectrometer with the pre-sample Bragg focusing optics found on a triple-axis spectrometer [43]. 
HYPSEC views the coupled cryogenic hydrogen moderator, and uses a combination of a Fermi chopper (acts as the monochromator), Bragg focusing optics and a moveable detector bank to select an energy window and vary the energy resolution at the sample position by almost an order of magnitude [43]. The SAC input is a single-energy representative integral neutron flux at $15 \mathrm{meV}$ that was simulated by B. L. Winn with McStas using $100 \%$ para hydrogen for the moderator. The SAC integral flux is the peak integral flux for the HYSPEC instrument. Iverson's measurement, shown in Fig. 20, was measured with a gold foil at the sample position and normalized to a $6 \%$ energy resolution at $15 \mathrm{meV}$ [44]. Stone's integrated beam monitor intensity (shown in Fig. 23) has been normalized to proton charge accumulated on the target, beam power, sample area, and Winn's measured energy resolution (shown in Fig. 22) [20]. It should be noted that Stone's spectrum (shown in Fig. 20) was measured with a calibrated beam monitor located just before the sample position [20]. Winn's measurement, shown in Fig. 20, was acquired by measuring the relative scattered flux from a $6 \mathrm{~mm}$ diameter vanadium rod (shown as HOPG in Fig. 21), and the relative scattered flux was scaled to a known neutron flux value (provided by Stone's previously measured spectrum) at $15 \mathrm{meV}[20,43]$. Winn's measurement was also normalized to the measured 180 $\mathrm{Hz}$ energy resolution shown in Fig. 22 [43]. The Granroth simulated neutron flux shown in Fig. 20 was calculated using McStas models [6, 45]. These McStas models incorporated HYSPEC's curved ballistic guide, the frame defining choppers, an energy-definition chopper, a focusing pyrolitic graphite monochromator, and a large detector positioned $4.5 \mathrm{~m}$ downstream of the sample position $[6,45]$. Granroth's raw neutron flux spectrum is shown in Fig. 24. Granroth's simulated neutron flux spectrum (shown in Fig. 20) was normalized to $2 \mathrm{~cm} \times 2 \mathrm{~cm}$ beam area at the sample position [6, 45]. The uniform upward skew in Granroth's spectrum may be attributed to the McStas model using a preliminary moderator source term $[6,45]$. The discrepancies between the SAC input neutron flux spectrum and the measured spectra shown in Fig. 20 may be a result of a different ortho to para hydrogen ratio in the moderator during the measurements than in the original SAC McStas simulation. 


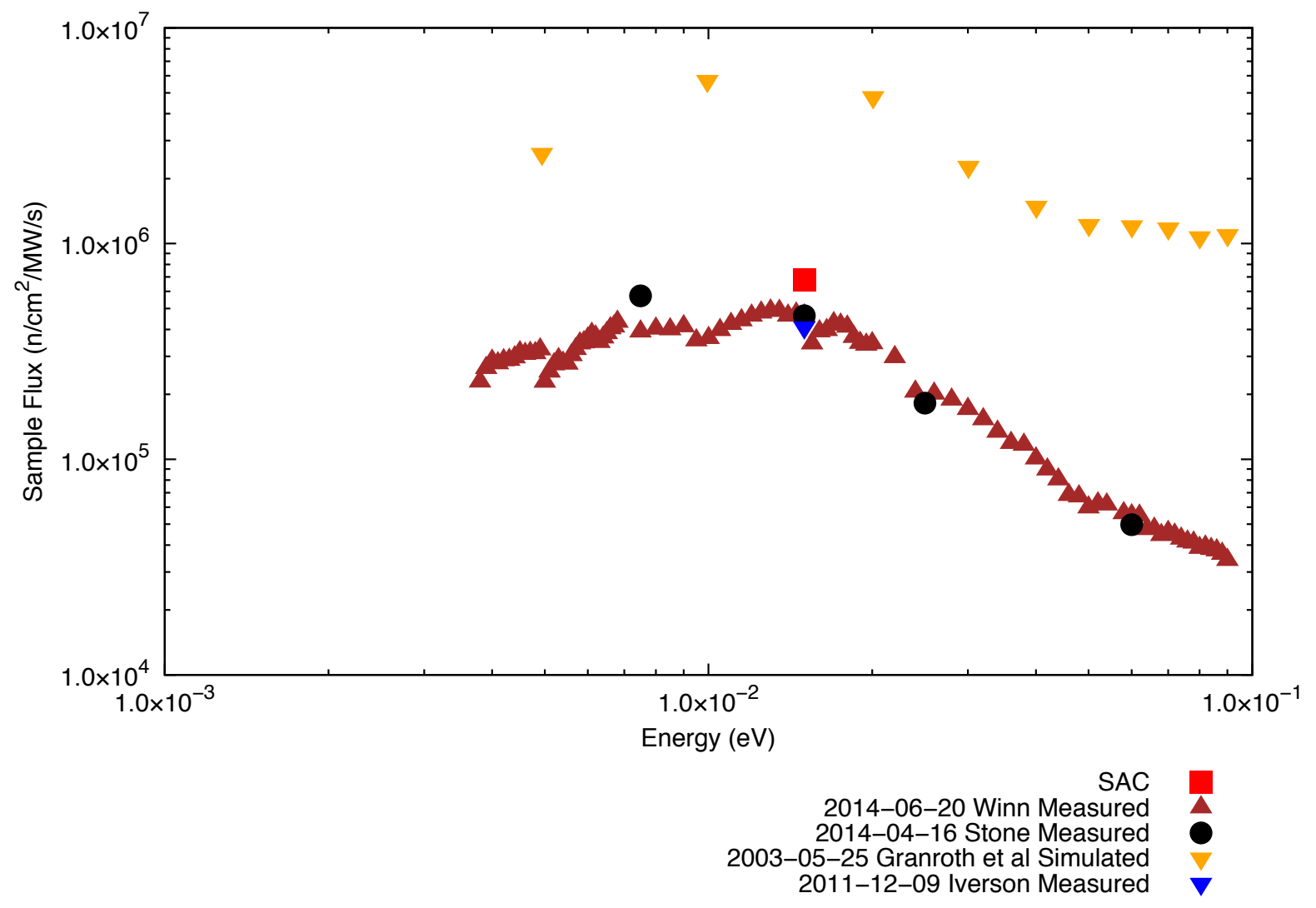

Fig. 20. HYSPEC Neutron Flux Comparison 


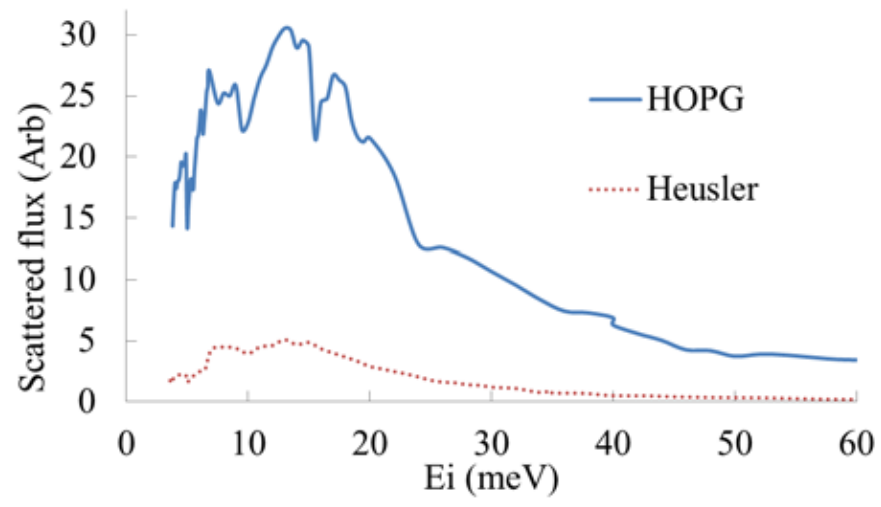

Fig. 21. HYSPEC Measured Neutron Flux by Winn [43]

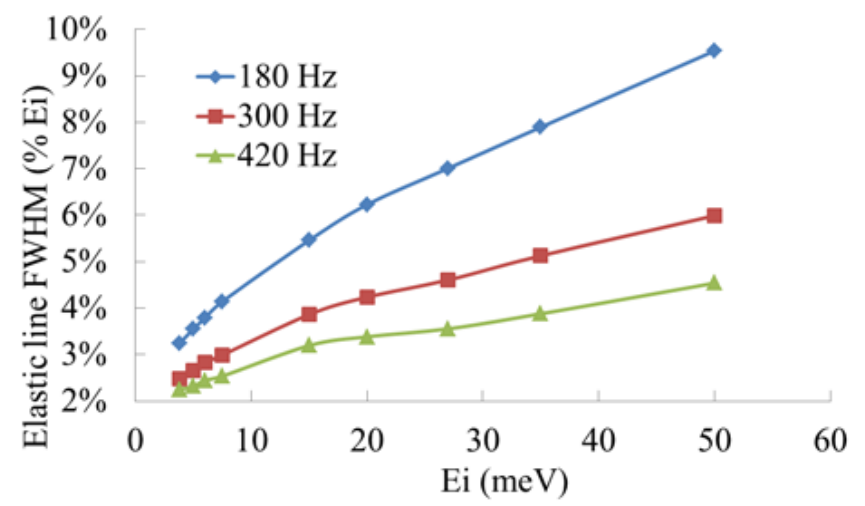

Fig. 22. HYSPEC Measured Energy Resolution by Winn [43] 


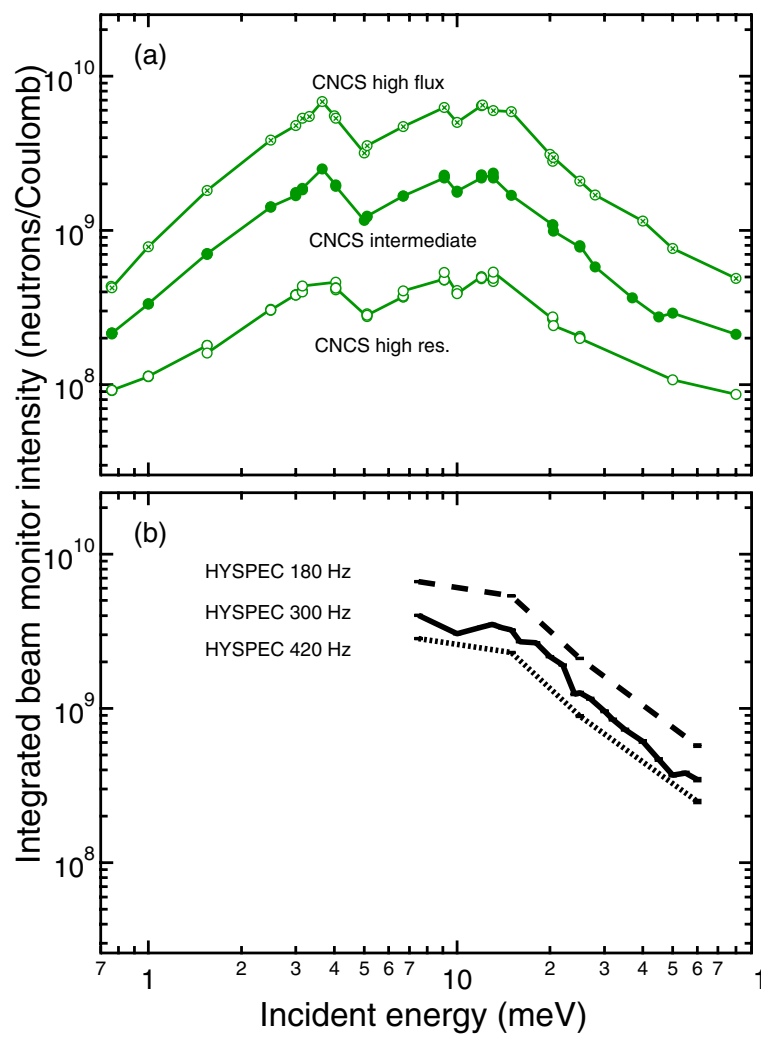

Fig. 23. HYSPEC Measured Neutron Flux as reported by Stone [20] 


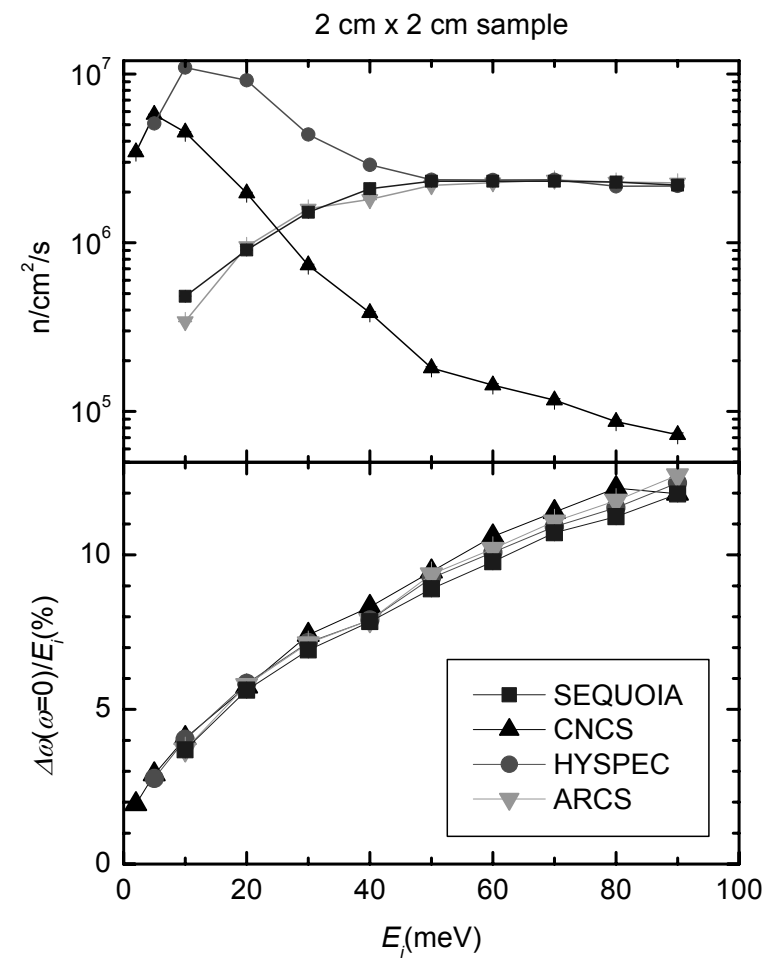

Fig. 24. HYPSEC Simulated Energy Flux and Energy Resolution calculated by Granroth et al $[6,45]$.

The Neutronics Analysis Team implements no change in neutron flux spectrum from the previous version of SAPEU in SAPEU (1.1.3) for the HYSPEC instrument because the integral flux that is currently in SAC provides a suffieciently convervative estimate of the neutron activation when compared to the other measurments in Fig. 20, and documents that the neutron flux in SAPEU (1.1.2) and (1.1.3) is B.L. Winn's $100 \%$ para hydrogen McStas simulation at $15 \mathrm{meV}$.

\subsection{BEAMLINE 15: NSE}

The NSE instrument is the spin-echo spectrometer at the SNS [46]. Fig. 25, Fig. 26, and Fig. 27 show the comparison between the SAC input neutron flux spectrum and Iverson's measured neutron flux spectrum [47] for the three different bender options offered by NSE. Iverson's spectrum was measured with a calibrated beam monitor placed at the sample position (17.34 m from the moderator) while SNS operated in a $5 \mathrm{~Hz}$ operational mode [47]. During the measurement, all of the choppers were parked open [47]. Iverson's measured spectrum has been normalized to the average beam power while taking the measurement and the beam area at the sample position. 


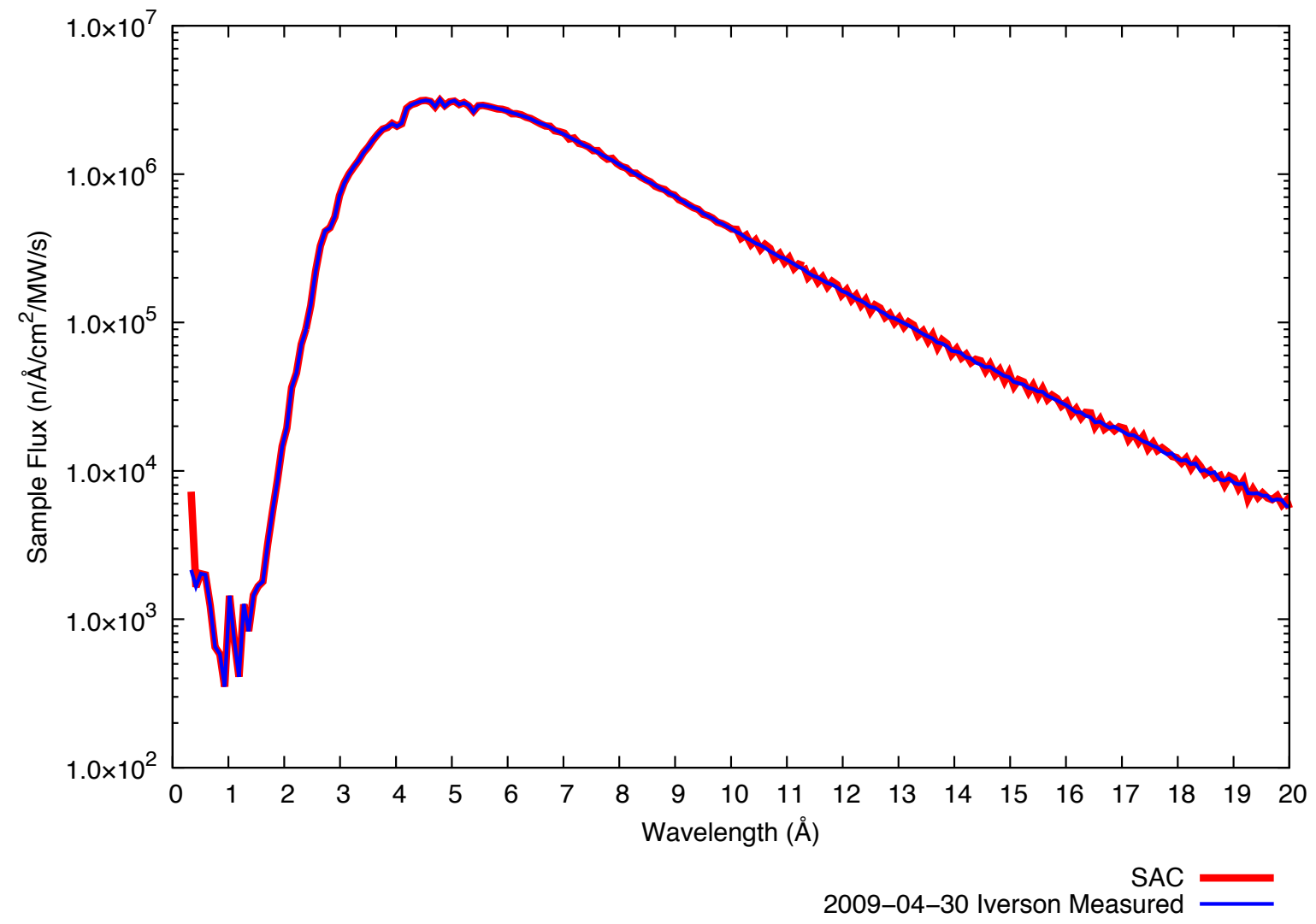

Fig. 25. NSE 23 mm Bender Wavelength Flux Comparison 


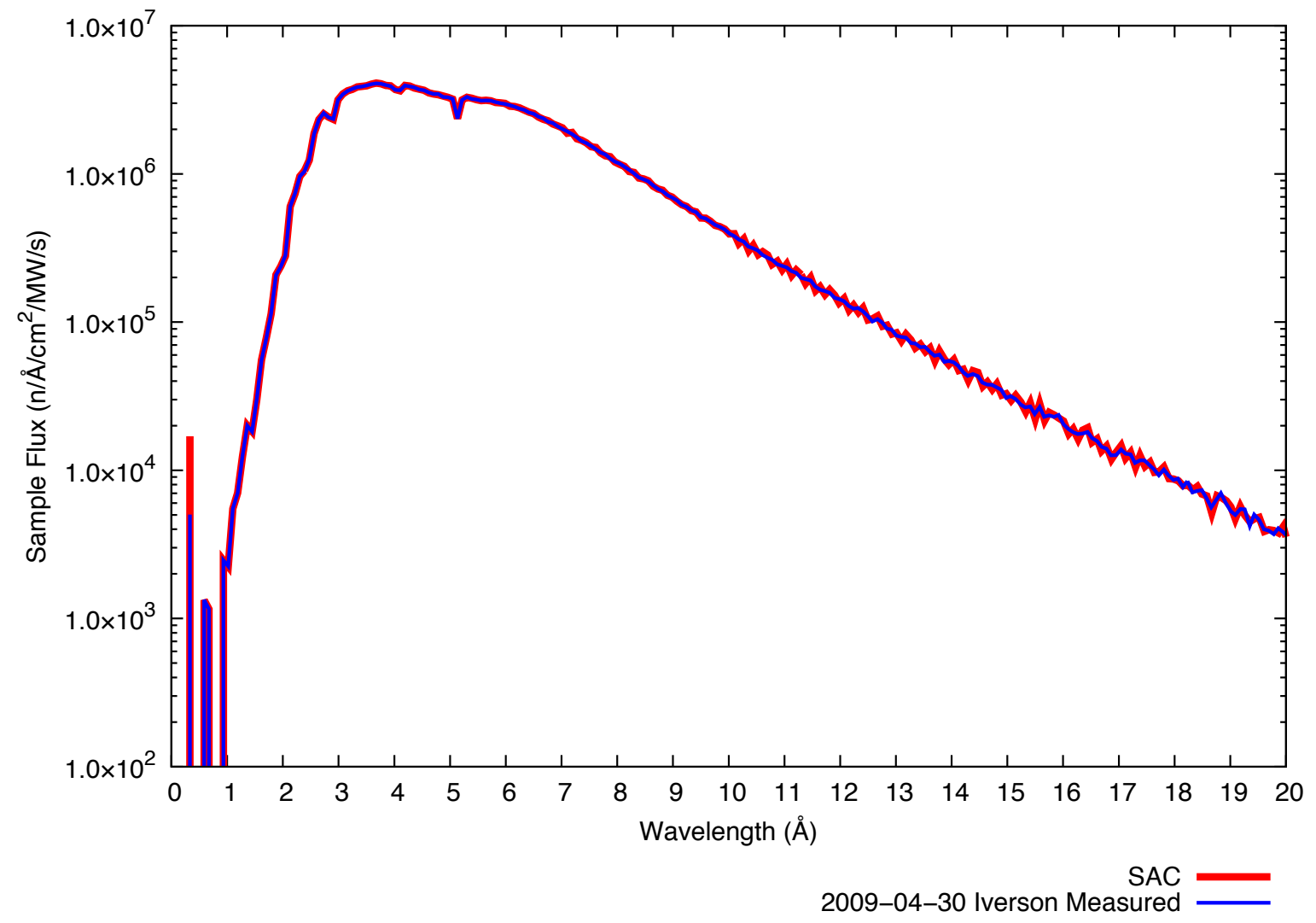

Fig. 26. NSE $60 \mathrm{~mm}$ Bender Wavelength Flux Comparison 


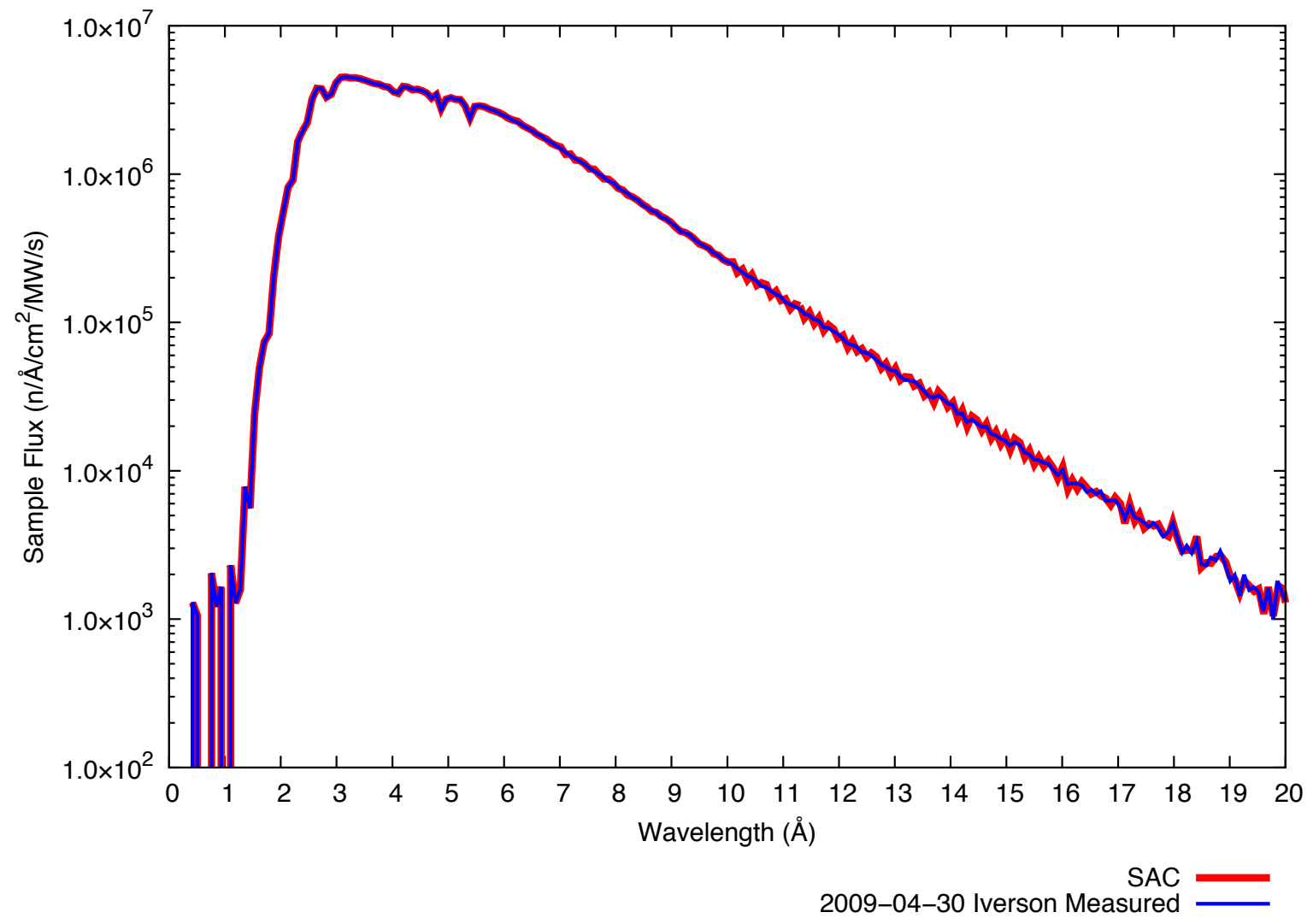

Fig. 27. NSE $120 \mathrm{~mm}$ Bender Wavelength Flux Comparison

Due to the two spectra in each figure (Fig. 26, Fig. 26, and Fig. 27) appearing to be from the same measurement, the Neutronics Analysis Team implements no change in the neutron flux spectrum from the previous version of SAPEU in SAPEU (1.1.3) for the NSE instrument, and documents that the input neutron flux spectrum to SAC is Iverson's measured spectrum [47].

\subsection{BEAMLINE 16A}

This beamline is currently not in the SAC.

\subsection{BEAMLINE 16B: VISION}

The VISION instrument is a crystal-analyzer spectrometer at the SNS [48]. Fig. 28 shows the comparison between the SAC input neutron flux spectrum and Gallmeier's simulated neutron flux spectrum for the VISION instrument [49]. Gallmeier uses an asbuilt McStas model to calculate the spectrum shown below at the sample position with an area of $15 \mathrm{~cm}^{2}$ [49]. A report of the details of the simulation is currently being written. 


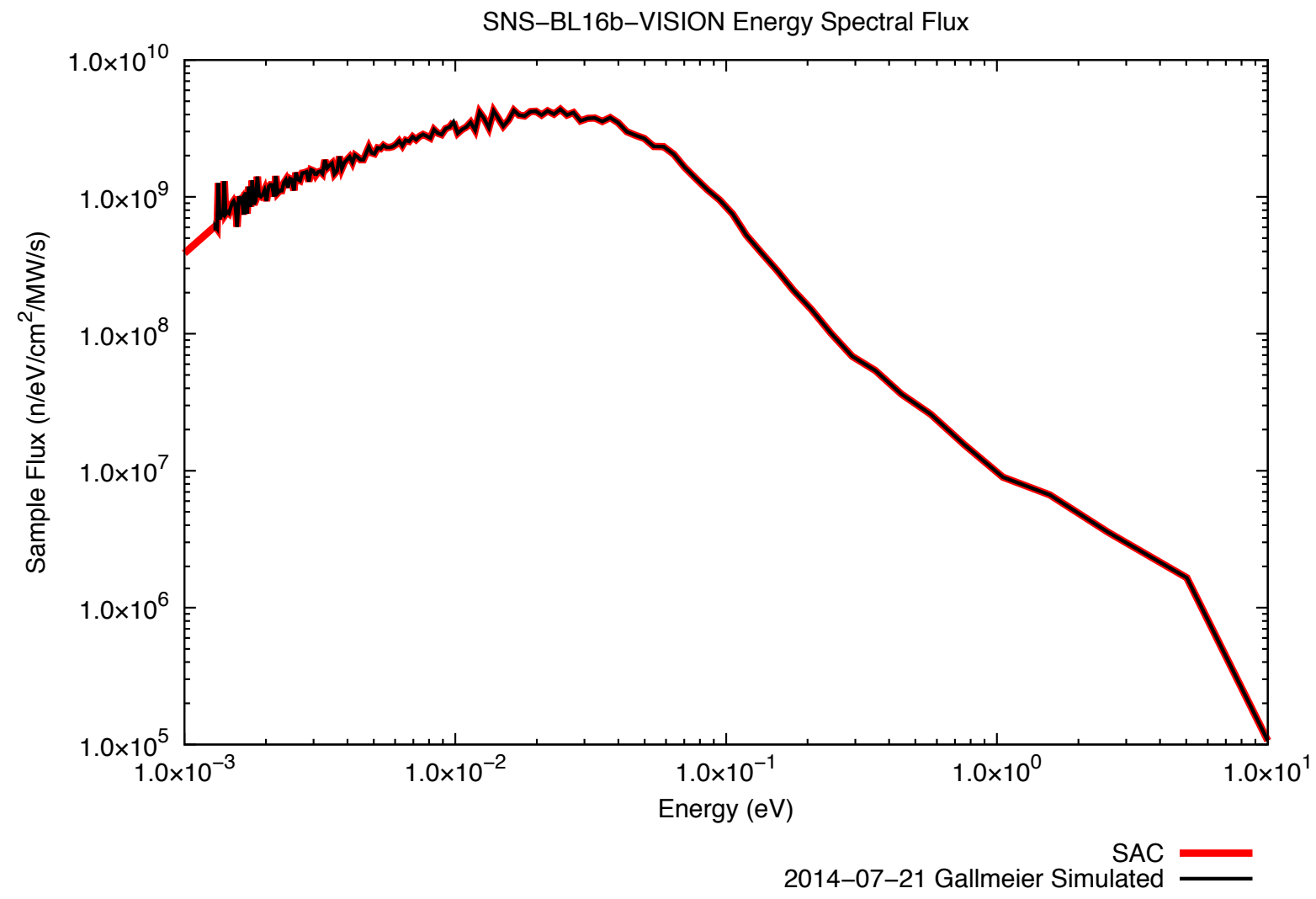

Fig. 28. VISION Energy Flux Comparison

Due to the two spectra in Fig. 28 appearing to be from the same simulation, the Neutronics Analysis Team implements no change in the neutron flux spectrum from the previous version of SAPEU in SAPEU (1.1.3), and documents that the SAC input neutron flux spectrum for VISION is Gallmeier's simulated neutron flux spectrum [49].

\subsection{BEAMLINE 17: SEQUOIA}

The SEQUOIA instrument is a fine resolution Fermi chopper spectrometer at the SNS [50]. Fig. 29 shows the SAC neutron flux spectrum compared with two simulated spectra and one measured spectrum. The origins of the SAC spectrum are unknown. The Debeer-Schmitt simulated neutron flux spectrum uses a McStas beamline model to simulate the neutron flux at the sample position, and this spectrum has been 
normalized by beam area, beam power, and energy bin width [51] disregarding chopper effects. It should be noted that the Debeer-Schmitt simulated neutron flux spectrum shown in Fig. 29 results from the base McStas model (documented in [52]) that Debeer-Schmitt updated with the latest SEQUOIA component configuration [53]. The Granroth simulated flux spectrum was simulated with a preliminary McStas model that included one of the first moderator source terms, and is expected to be lower in neutron flux magnitude $[6,45]$. The Granroth simulated neutron flux spectrum was calculated by simulating a $4 \mathrm{~cm} \times 4 \mathrm{~cm}$ beam at the sample position with the Fermi chopper spinning at $600 \mathrm{~Hz}$, and then placing a detector $3 \mathrm{~m}$ downstream of the sample position, while changing the phasing of the Fermi chopper to allow different wavelength bands to pass through the chopper (raw spectrum shown in Fig. 30) [6, 45]. This spectrum was then normalized to the beam area at the sample position, beam power, and the energy resolution shown in Fig. 30 [6, 45]. Stone's spectrum was measured with a calibrated beam monitor located just before the sample position. The integrated beam monitor intensity, shown in Fig. 31 (c), was normalized to proton charge accumulated on the target, beam power, sample area, and the energy resolution shown in Fig. 32 (c) [20]. It should be noted that Stone's neutron flux spectrum shown in Fig. 29 is composed of a spectrum obtained with the $100 \mathrm{meV}$ chopper spinning at $300 \mathrm{~Hz}$ applied from 0.0 to $0.1 \mathrm{eV}$, and a spectrum obtained with the $700 \mathrm{meV}$-chopper spinning at $600 \mathrm{~Hz}$ applied from 0.1 to $1.0 \mathrm{eV}$ as shown in Fig. 31 (c) [20]. The two spectral components were normalized with their corresponding energy resolution shown in Fig. 32 (c). It should also be noted that the Debeer-Schmitt simulation is calculated without the Fermi Chopper in the beam, while the other two spectra are measured and simulated with the Fermi Chopper. 


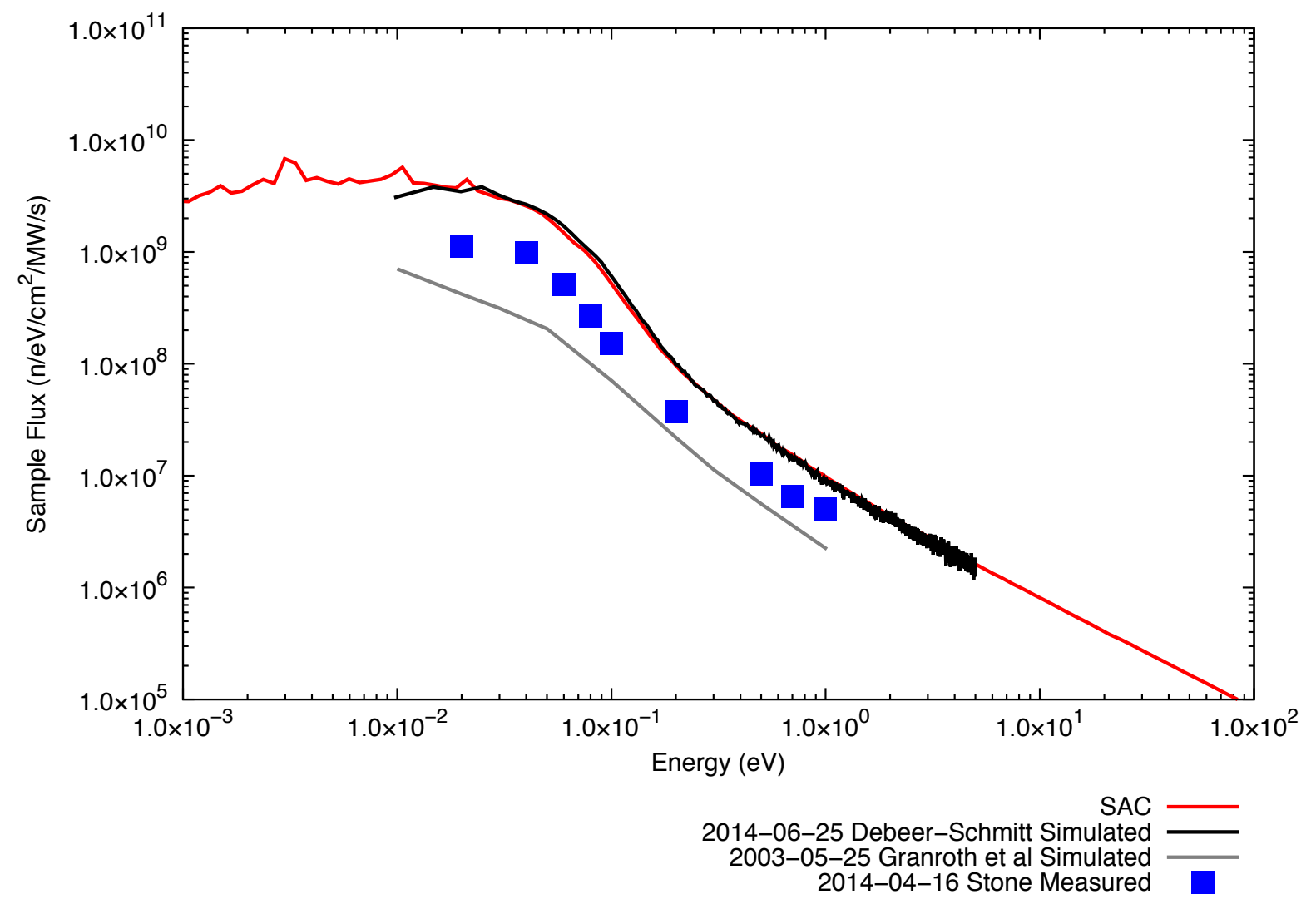

Fig. 29. SEQUOIA Neutron Flux Comparison 


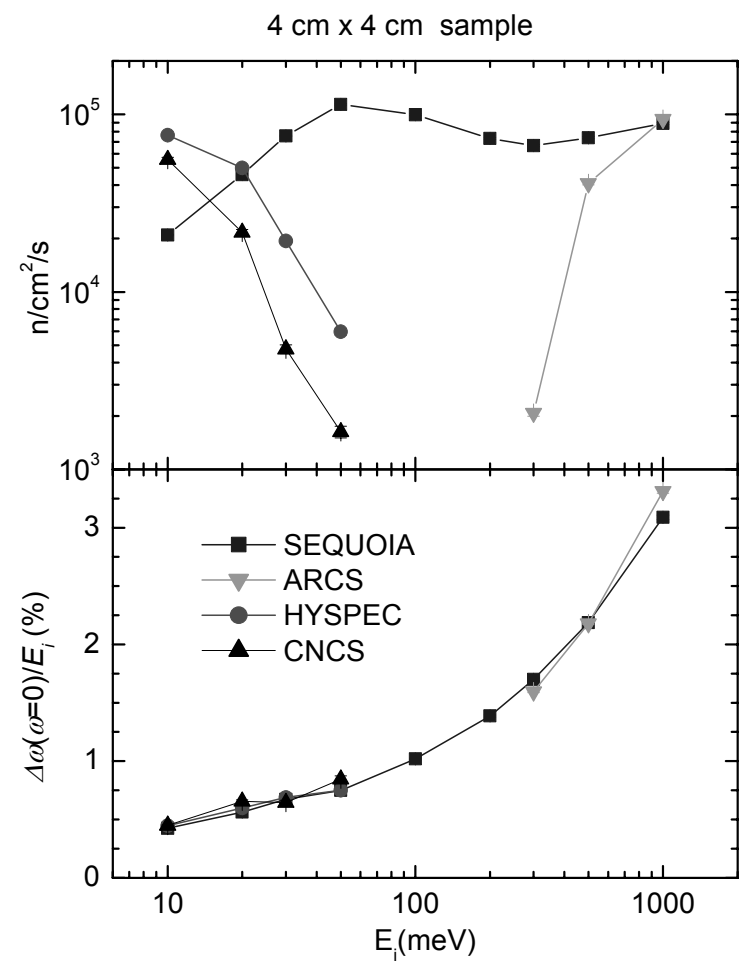

Fig. 30. SEQUOIA Neutron Flux Simulated by Granroth et al $[6,45]$ 

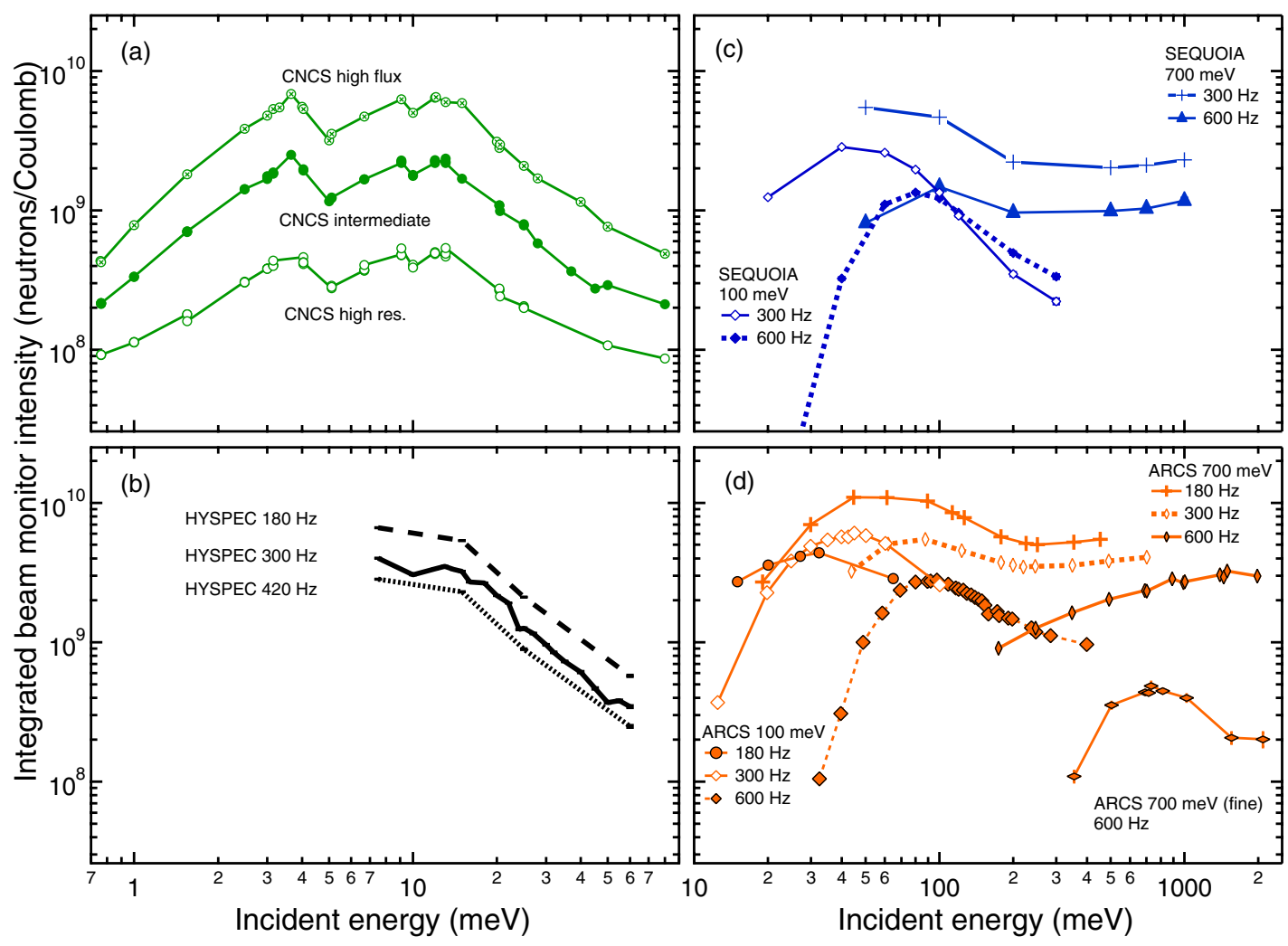

Fig. 31. Chopper Spectrometers Neutron Flux Measured by Stone [20] 


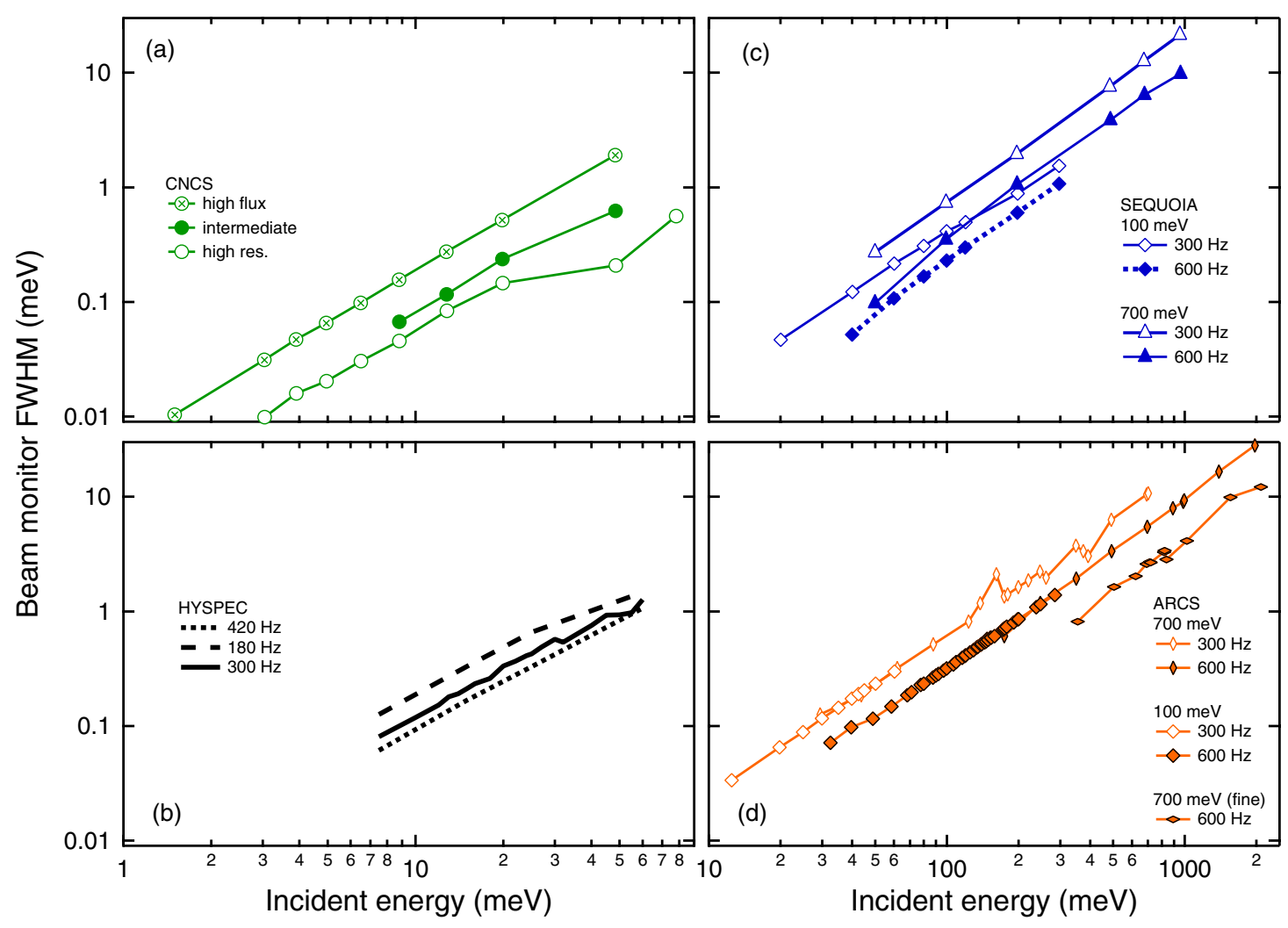

Fig. 32. Chopper Spectrometers Energy Resolution Measured by Stone [20]

Based on the comparison made in Fig. 29, the Neutronics Analysis Team implements no change in the neutron flux spectrum from the previous version of SAPEU in SAPEU (1.1.3), and documents that the input neutron flux spectrum to SAC is DeBeer-Schmitt's simulated spectrum for the SEQUOIA instrument [53]. With the Granroth simulated spectrum and Stone measured spectrum within a factor of 2 to 5 , changing the spectrum could not be justified $[20,45]$. The spectra simulated with the white beam mode would provide a conservative estimate for the sample activation.

\subsection{BEAMLINE 18: ARCS}

ARCS is a wide Angle-Range Chopper Spectrometer at the SNS [54]. Fig. 33 shows the SAC neutron flux spectrum compared with two simulated spectra and one measured spectrum. The origins of the SAC spectrum are unknown. The 2003 Abernathy simulated flux spectrum was simulated with a preliminary McStas model that included one of the first decoupled water moderator source terms, and is expected to be lower in neutron flux magnitude [6, 45]. The 2003 Abernathy simulated neutron flux spectrum was calculated by simulating a $4 \mathrm{~cm} \times 4 \mathrm{~cm}$ beam at the sample position with the Fermi chopper spinning at $600 \mathrm{~Hz}$ with $2 \mathrm{~mm}$ slits (the curvature of the chopper blades was optimized at each simulated energy), and then placing a detector $3 \mathrm{~m}$ downstream of the sample position (raw spectrum shown in Fig. 34) [6, 45]. This spectrum was then normalized to the beam area at the sample position, beam power, and the energy resolution shown in Fig. 34 [6, 45]. The 2010 Abernathy simulated neutron flux spectrum uses MCNPX to simulate the neutron flux at the sample position, and this spectrum has been normalized by beam area, beam power, and guide gain factors $[52,55]$. Stone's spectrum was measured with a calibrated beam monitor located just before the sample position, and the integrated beam monitor intensity, shown in Fig. 
35 (d), has been normalized to proton charge accumulated on the target, beam power, sample area, and the energy resolution shown in Fig. 36 (d) [20]. It should be noted that Stone's neutron flux spectrum shown in Fig. 35 is a combination of the spectrum noted as the $100 \mathrm{meV}$ chopper at $180 \mathrm{~Hz}$ from 0.015 to $0.032 \mathrm{eV}$ and $300 \mathrm{~Hz}$ from 0.034 to $0.06 \mathrm{eV}$ shown in Fig. 35 (d), and the spectrum noted as the $700 \mathrm{meV}$ chopper at $300 \mathrm{~Hz}$ from 0.087 to $0.7 \mathrm{eV}$ and $600 \mathrm{~Hz}$ from 0.89 and above shown in Fig. 35 (d) [20]. The combination of the four spectra have been normalized to their corresponding energy resolution spectra shown in Fig. 36 (d). The Abernathy-measured neutron flux spectrum was measured with Monitor 1 on ARCS, which is $1.77 \mathrm{~m}$ upstream of the sample position, the Fermi choppers has been translated out of the beam, and with the T0 chopper stopped in the open position [56]. The Abernathy measured and 2010 simulated neutron flux spectra are in good agreement.

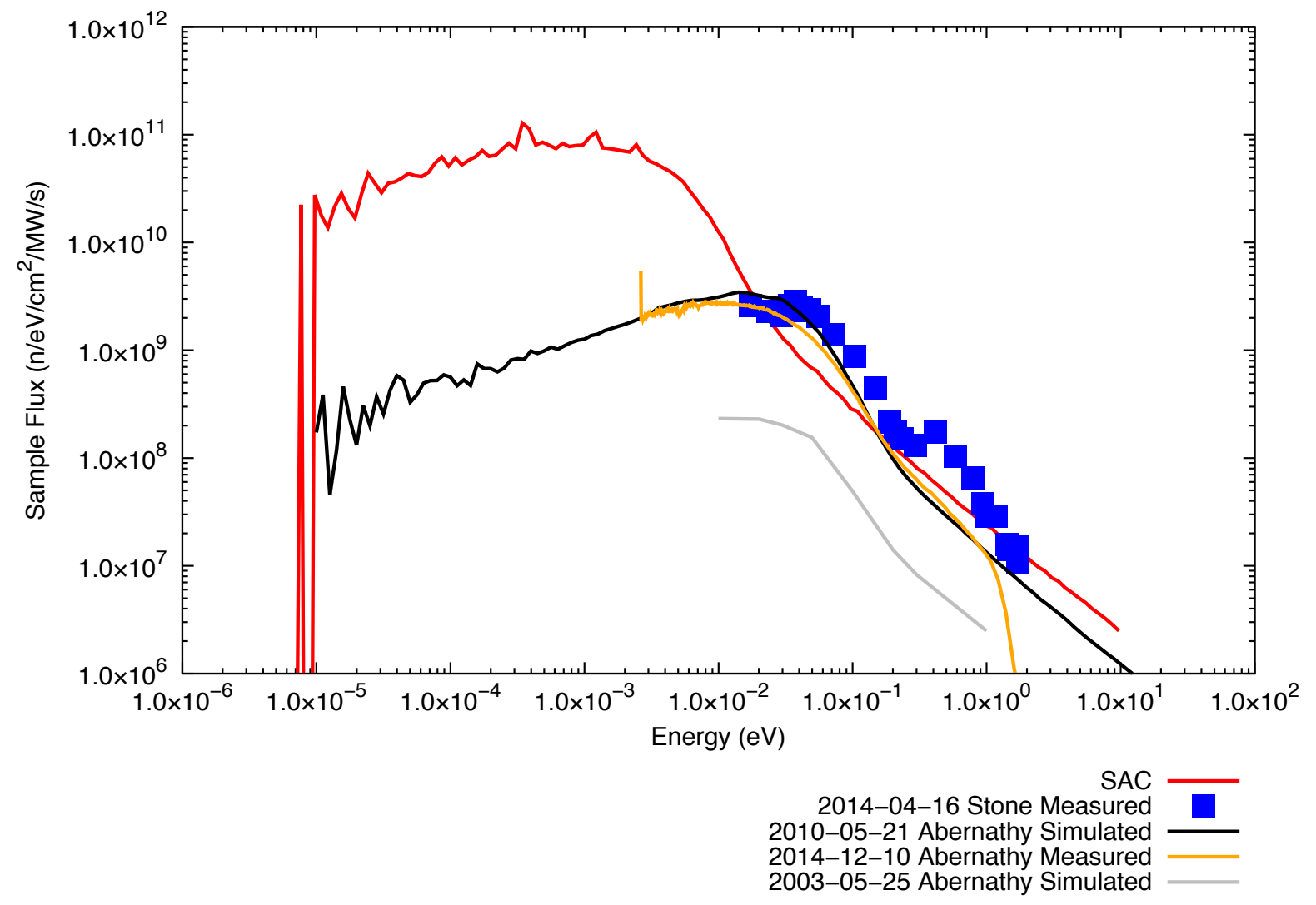

Fig. 33. ARCS Neutron Flux Comparison 


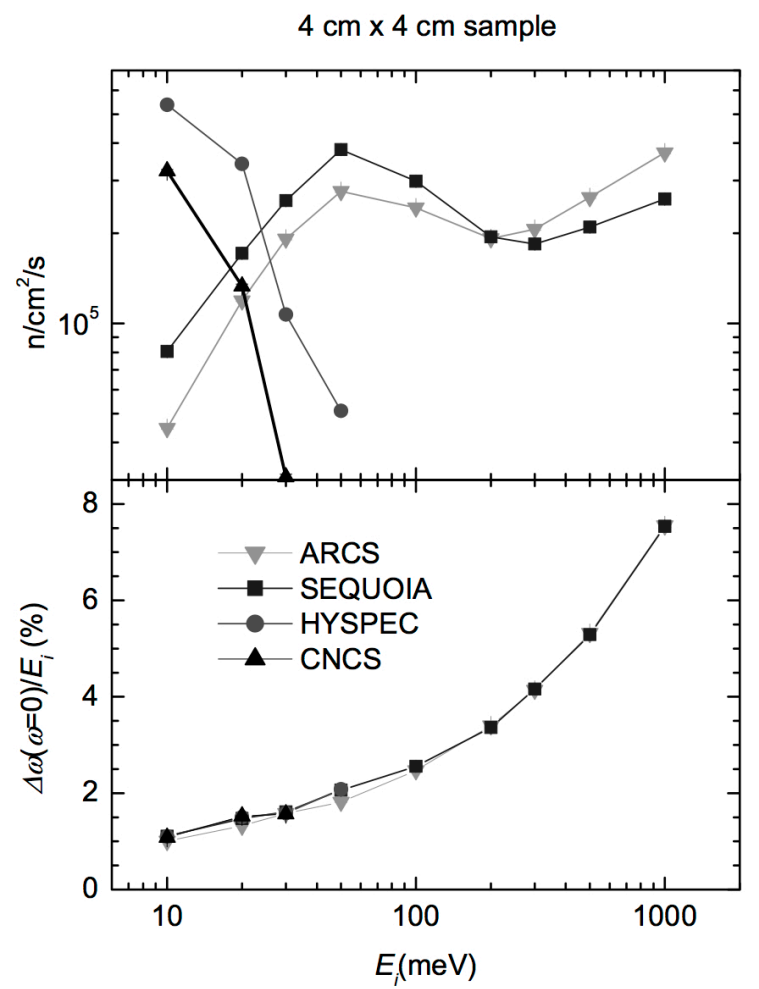

Fig. 34. ARCS Neutron Flux Simulated by Abernathy et al $[6,45]$ 

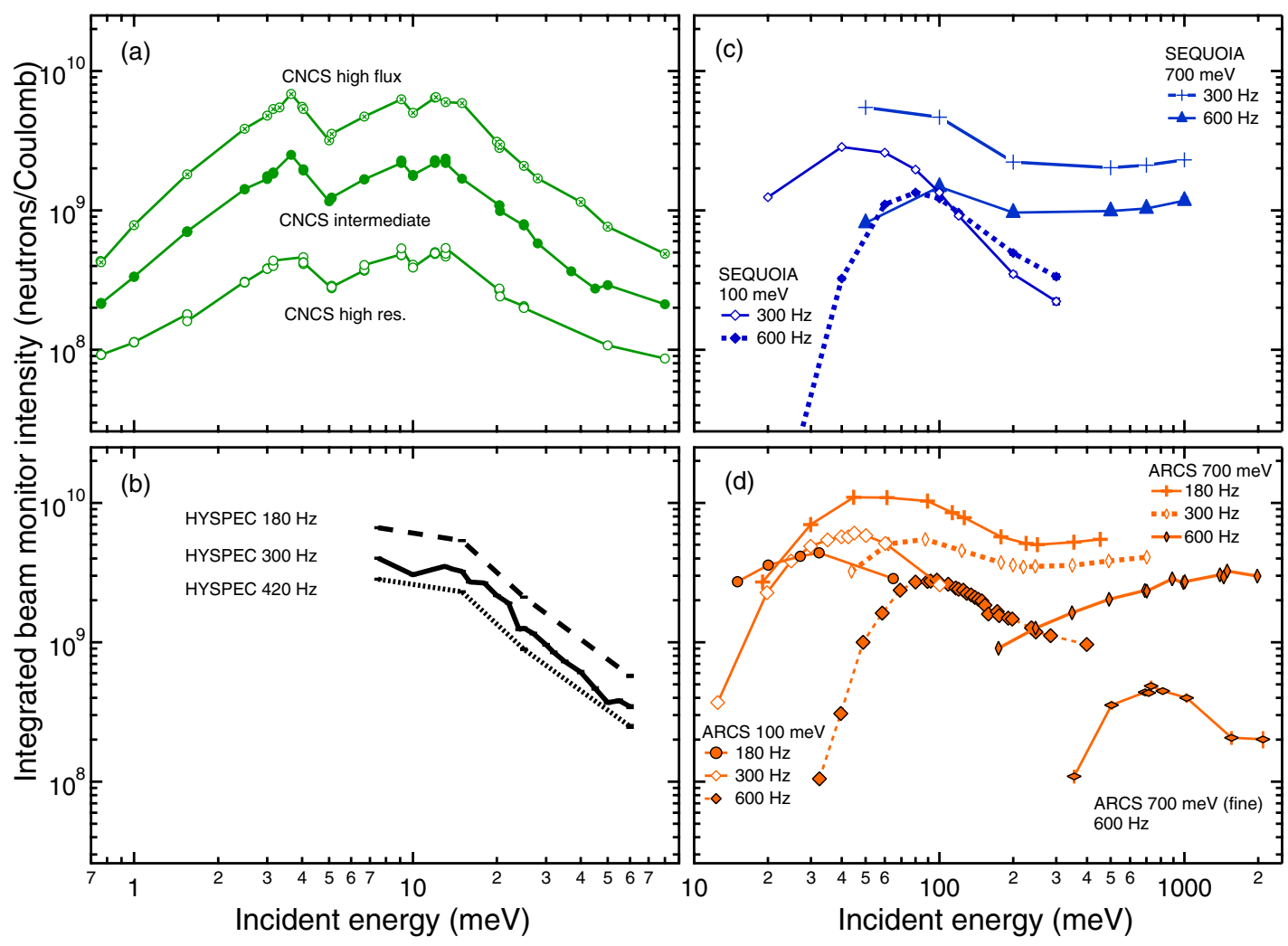

Fig. 35. Chopper Spectrometers Neutron Flux Measured by Stone [20] 


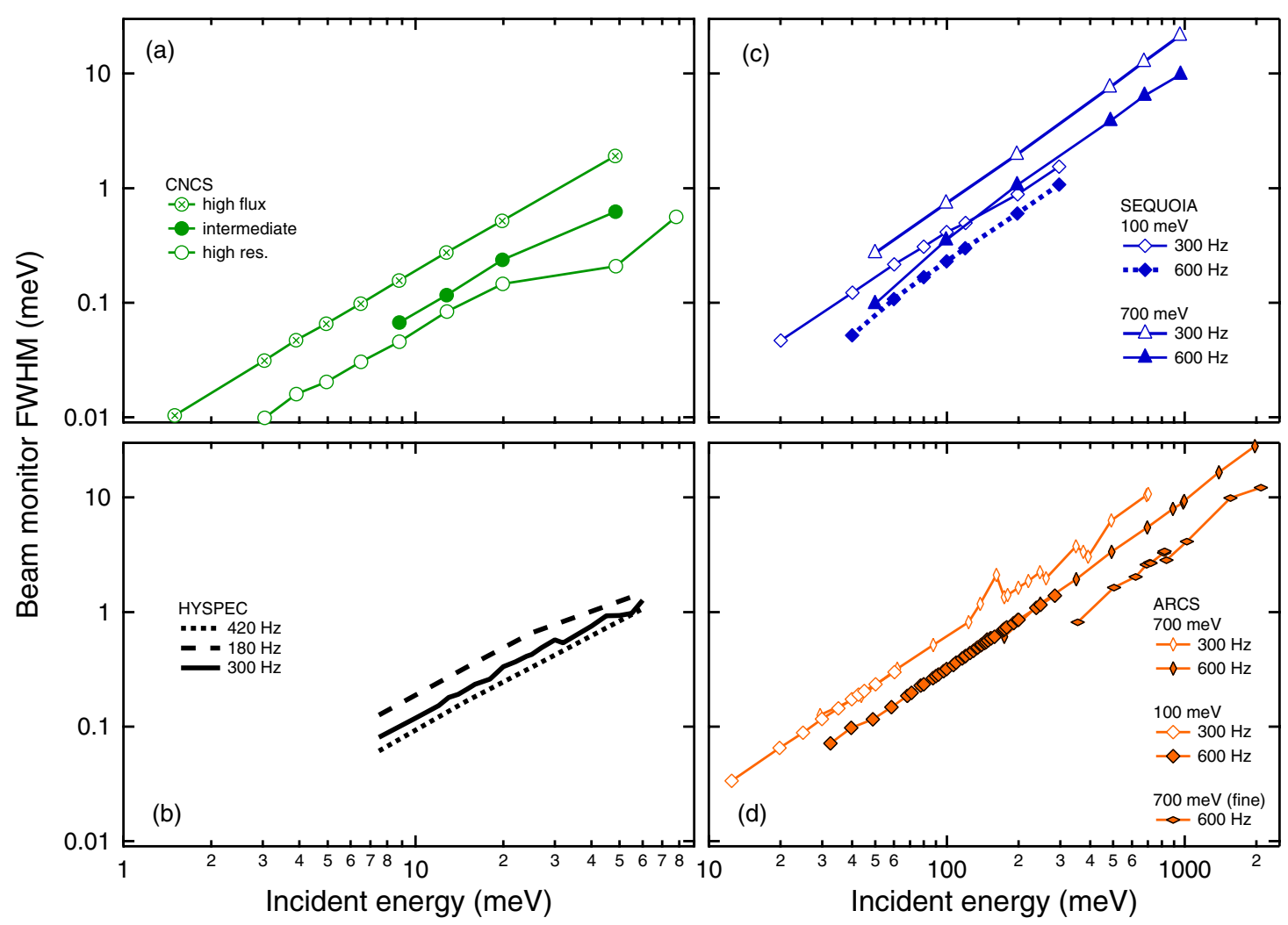

Fig. 36. Chopper Spectrometers Energy Resolution Measured by Stone [20]

Based on the comparison made in Fig. 33, the Neutronics Analysis Team implements the 2010 Abernathy simulated spectrum in SAPEU (1.1.3) because of its good agreement with the Abernathy measured spectrum, and because the simulation covers a larger energy range $[52,55]$.

\section{SUMMARY}

We have described the selection of spectral intensities for the SAPEU program to best describe the activation of samples on SNS beamlines. Where there is question between different spectral intensities, we recommend using the more conservative (higher) values. These spectra will be implemented in version (1.1.3) of the SAPEU code, to address one of the three major categories of error identified in the SAPEU / SAC code system by our validation exercise documented elsewhere [3]. Table 1 below is a summary of the implemented spectra for each beamline. 
Table 1: Summary of SAC Neutron Flux Recommendations

\begin{tabular}{|c|c|c|c|}
\hline Beamline & $\begin{array}{c}\text { Updated in } \\
\text { SAPEU (1.1.3) } \\
\text { (Yes/No) }\end{array}$ & Source of Spectrum & $\begin{array}{c}\text { Ratio of Total } \\
\text { Neutron Flux } \\
\text { (current/previous) }\end{array}$ \\
\hline BL1b-NOMAD & No & Simulated by F.X. Gallmeier [5] & $\mathrm{N} / \mathrm{A}$ \\
\hline BL2-BASIS & Yes & Measured by E. Mamontov [7] & 1.98 \\
\hline BL3-SNAP & No & Simulated by P.D. Ferguson & N/A \\
\hline BL4a-MR-col & Yes & Scaled to the measurement by V. Lauter [13] & 0.0213 \\
\hline BL4b-MR-col & Yes & Scaled to the measurement by E.B. Iverson [16] & 0.0781 \\
\hline BL5-CNCS & Yes & Measured by M.B. Stone [20] & 0.0211 \\
\hline BL6-EQSANS & Yes & $\mathbf{1 0 0 \%}$ para H Simulated by F.X. Gallmeier [22] & 0.07 \\
\hline BL7-VULCAN & Yes & Simulated by F.X. Gallmeier [5] & 0.66 \\
\hline BL9-CORELLI & Yes & Measured by E.B. Iverson [27] & N/A \\
\hline $\begin{array}{l}\text { BL11a-POWGEN- } \\
\text { HighIntensity }\end{array}$ & Yes & Simulated by F.X. Gallmeier [5] & 0.648 \\
\hline $\begin{array}{l}\text { BL11a-POWGEN- } \\
\text { HighResolution }\end{array}$ & Yes & Simulated by J.P. Hodges [30] & 0.547 \\
\hline BL11b-MANDI & Yes & Simulated by F.X. Gallmeier [33] & N/A \\
\hline BL12-TOPAZ & Yes & Simulated by X. Wang [37] & $1.603(\mathrm{E}<1 \mathrm{eV})$ \\
\hline BL13-FNPB & Yes & Measured by E.B. Iverson [41] & 0.99 \\
\hline BL14b-HYSPEC & No & Simulated by B.L.Winn & N/A \\
\hline BL15-NSE & No & Measured by E.B. Iverson [47] & N/A \\
\hline BL16b-VISION & No & Simulated by F.X. Gallmeier [49] & N/A \\
\hline BL17-SEQUOIA & No & Simulated by Debeer-Schmitt [53] & $\mathrm{N} / \mathrm{A}$ \\
\hline BL18-ARCS & Yes & 2010 Simulated by D.L. Abernathy [55] & 0.401 \\
\hline
\end{tabular}




\section{REFERENCES}

[1] J. M. Carpenter, "Pulsed Spallation Neutron Sources for Slow-Neutron Scattering," Nuclear Instruments \& Methods, vol. 145, pp. 91-113, 1977.

[2] W. Lu, P. D. Ferguson, F. X. Gallmeier, E. B. Iverson, I. I. Popova, and Y. Wang, "A Sample Activation Program for Neutron-Scattering Experiments," Nuclear Technology, vol. 168, pp. 970976, 2009.

[3] T. C. McClanahan, E. B. Iverson, and F. X. Gallmeier, "SAC Activation Analysis and Validation," Spallation Neutron Source, Oak Ridge National LaboratorySNS-ISDD-NSD-TR002-R002 2015.

[4] J. Neuefeind, M. Feygenson, J. Carruth, R. Hoffmann, and K. K. Chipley, "The Nanoscale Ordered MAterials Diffractometer NOMAD at the Spallation Neutron Source SNS," Nuclear Instruments and Methods in Physics Research Section B: Beam Interactions with Materials and Atoms, vol. 287, pp. 68-75, Aug. 2012.

[5] A. C. Hamilton and F. X. Gallmeier, "A Suite of SNS Neutron Instrument Models," in Tenth International Topical Meeting on Nuclear Applications of Accelerators, Knoxville, TN, 2011.

[6] E. B. Iverson, P. D. Ferguson, F. X. Gallmeier, and I. I. Popova, "Detailed SNS neutronics calculations for scattering instrument design: SCT configuration," 2002.

[7] E. Mamontov and K. W. Herwig, "A time-of-flight backscattering spectrometer at the Spallation Neutron Source, BASIS," Review of Scientific Instruments, vol. 82, pp. 085109-1-10, 2011.

[8] C. Tulk. (2014, September 30). Spallation Neutrons and Pressure Diffractometer at SNS [Online]. Available: http://neutrons.ornl.gov/snap/

[9] E. B. Iverson. Email. From: iversoneb@ornl.gov To: mcclanahantc@ornl.gov Oak Ridge National Laboratory. July 82014.

[10] P. D. Ferguson. From: fergusonpd@ornl.gov To: luw2@ornl.gov Oak Ridge National Laboratory. 2008.

[11] H. Ambaye, R. Goyette, A. Parizzi, and F. Klose, "SNS Magnetism Reflectometer," Neutron News, vol. 19, pp. 11-13, July 282008.

[12] F. Klose, "Design Criteria for the Spallation Neutron Source Instrument Systems Magnetism Reflectometer," Oak Ridge National Laboratory, Spallation Neutron SourceSNS 107050000DC0001-R01 Oct. 312004.

[13] V. Lauter. Email. From: lauterv@ornl.gov To: mcclanahantc@ornl.gov Oak Ridge National Laboratory. June 192014.

[14] V. Lauter. Meeting. From: V. Lauter To: T. C. McClanahan Oak Ridge National Laboratory. Aug. 262014.

[15] J. F. Ankner, X. Tao, C. E. Halbert, J. F. Browning, S. Michael Kilbey, O. A. Swader, et al., "The SNS Liquids Reflectometer," Neutron News, vol. 19, pp. 14-16, July 2008.

[16] E. B. Iverson, "Assessment of Dead-Time Effects in SNS Neutronics Beam Characterization Apparatus," ORNL, SNSSep. 2008.

[17] G. Ehlers, A. A. Podlesnyak, J. L. Niedziela, E. B. Iverson, and P. E. Sokol, "The new cold neutron chopper spectrometer at the Spallation Neutron Source: Design and performance," Review of Scientific Instruments, vol. 82, pp. 1-6, Aug 2011.

[18] E. B. Iverson. Email. From: iversoneb@ornl.gov To: mcclanahantc@ornl.gov Oak Ridge National Laboratory. July 82014.

[19] E. B. Iverson, M. B. J., B. D. V., R. Cooper, P. D. Ferguson, D. W. Freeman, et al., "Neutronic Measurements to Commission the SNS," in International Collaboration of Advanced Neutron Sources (ICANS-XVII), 2006, p. 436.

[20] M. B. Stone, J. L. Niedziela, D. L. Abernathy, L. DeBeer-Schmitt, G. Ehlers, O. Garlea, et al., "A comparison of four direct geometry time-of-flight spectrometers at the Spallation Neutron Source," Review of Scientific Instruments, vol. 85, pp. -, 2014. 
[21] J. K. Zhao, C. Y. Gao, and D. Liu, "The extended Q-range small-angle neutron scattering diffractometer at the SNS," Journal of Applied Crystallography, vol. 43, pp. 1068-1077, Oct. 2010.

[22] F. X. Gallmeier. Email. From: gallmeierfx@ornl.gov To: mcclanahantc@ornl.gov 5/1/14 2014.

[23] E. B. Iverson, "Flux Measurements on SNS BL06 EQ-SANS," Oak Ridge National LaboratoryJan. 2014.

[24] X. L. Wang, "Conceptual Design of the VULCAN Diffractometer," Oak Ridge National Laboratory2000.

[25] E. B. Iverson, A. D. Stoica, and W. Lu, "Intensity Gain from VULCAN Guide 9," Oak Ridge National LaboratoryOct. 2013.

[26] S. Rosenkranz and R. Osborn, "Corelli: Efficient single crystal diffraction with elastic discrimination," Pramana, vol. 71, pp. 705-711, 2008/10/01 2008.

[27] F. Ye. Email. From: yef1@ornl.gov To: luw2@ornl.gov Oak Ridge National Laboratory. Sep. 10 2014.

[28] A. Huq, J. P. Hodges, O. Gourdon, and L. Heroux, "Powgen: A third-generation high-resolution high-throughput powder diffraction instrument at the Spallation Neutron Source," in European Powder Diffraction Conference, Munich, Germany, 2011, pp. 127-135.

[29] E. B. Iverson, "Incident Beam Measurements on SNS BL11a POWGEN," Oak Ridge National LaboratoryNov. 2013.

[30] J. P. Hodges, "POWGEN Issues," unpublished.

[31] L. Coates, A. D. Stoica, C. Hoffmann, J. Richards, and R. Cooper, "The macromolecular neutron diffractometer (MaNDi) at the Spallation Neutron Source, Oak Ridge: enhanced optics design, high-resolution neutron detectors and simulated diffraction," Journal of Applied Crystallography, vol. 43, pp. 570-577, 2010.

[32] M. J. Frost. Email. From: frostmj@ornl.gov To: mcclanahantc@ornl.gov Oak Ridge National Laboratory. Dec. 42014.

[33] F. X. Gallmeier. Email. From: gallmeierfz@ornl.gov To: mcclanahantc@ornl.gov Oak Ridge National Laboratory. Dec. 052014.

[34] E. B. Iverson, "High-Bandwidth Beam Characterization for SNS BL11B; MaNDi," ORNL, SNS, Report Nov. 302012.

[35] E. B. Iverson. Email. From: iversoneb@ornl.gov To: mcclanahantc@ornl.gov Oak Ridge National Laboratory. Dec. 42014.

[36] M. Frost, C. Hoffmann, J. Thomison, M. Overbay, M. Austin, P. Carman, et al., "Initial testing of a Compact Crystal Positioning System for the TOPAZ Single-Crystal Diffractometer at the Spallation Neutron Source," Journal of Physics: Conference Series, vol. 251, p. 012084, 2010.

[37] X. Wang. Email. From: wangx@ornl.gov To: mcclanahantc@ornl.gov Oak Ridge National Laboratory. Oct. 172014.

[38] E. B. Iverson. Email. From: iversoneb@ornl.gov To: mcclanahantc@ornl.gov Oak Ridge National Laboratory. July 082014.

[39] N. Fomin, G. L. Greene, R. Allen, V. Cianciolo, C. Crawford, T. Ito, et al., "Fundamental Neutron Physics Beamline at the Spallation Neutron Source at ORNL," Nuclear Instruments and Methods in Physics Research Section A: Accelerators, Spectrometers, Detectors and Associated Equipment, vol. 773, pp. 45-51, Feb. 2015.

[40] P. R. Huffman, G. L. Greene, R. R. Allen, V. Cianciolo, R. R. Huerto, and P. Hoehler, "Beamline Perfomance Simulations for the Fundamental Neutron Physics Beamline at the Spallation Neurton Source," Journal of Research of the Natinal Institute of Standards and Technology, vol. 110, pp. 161-168, May-June 2005.

[41] E. B. Iverson, N. Fomin, R. G. Cooper, and D. W. Freeman, "Post-Repair Spectral Measurements on the SNS Bottom Downstream Coupled Hydrogen Moderator," Oak Ridge National LaboratoryApril 2009. 
[42] S. M. Shapiro, I. A. Zaliznyak, L. Passell, V. J. Ghosh, W. J. Leonhardt, and M. E. Hagen, "HYSPEC: A crystal time-of-flight hybrid spectrometer for the spallation neutron source with polarization capabilities," Physica B: Condensed Matter, vol. 385-386, Part 2, pp. 1107-1109, Nov. 152006.

[43] B. Winn, U. Filges, V. O. Garlea, M. Graves-Brook, M. Hagen, C. Jiang, et al., "Recent progess on HYSPEC, and its polarization analysis capabilities," EPJ Web of Conferences, 2014.

[44] E. B. Iverson. Email. From: iversoneb@ornl.gov To: mcclanahantc@ornl.gov Oak Ridge National Laboratory. July 082014.

[45] G. E. Granroth and D. L. Abernathy, "Performance Comparisons of Four Direct Geometry Spectrometers Planned for the Spallation Neutron Source," in 16th Meeting of the International Collaboration on Advanced Neutron Sources, Dusseldorf-Neuss, Germany, 2003.

[46] M. Ohl, M. Monkenbusch, N. Arend, T. Kozielewski, G. Vehres, C. Tiemann, et al., "The spinecho spectrometer at the Spallation Neutron Source (SNS)," Nuclear Instruments and Methods in Physics Research Section A: Accelerators, Spectrometers, Detectors and Associated Equipment, vol. 696, pp. 85-99, Dec. 222012.

[47] E. B. Iverson, N. Arend, T. C. Kozielewski, and M. E. Ohl, "Incident Beam Characterization for Neutron Spin Echo SNS Beam-Line 15," Oak Ridge National LaboratoryJuly 2011.

[48] P. A. Seeger, L. L. Daemen, and J. Z. Larese, "Resolution of VISION, a crystal-analyzer spectrometer," Nuclear Instruments and Methods in Physics Research Section A: Accelerators, Spectrometers, Detectors and Associated Equipment, vol. 604, pp. 719-728, June 112009.

[49] F.X. Gallmeier. Email. From: gallmeierfz@ornl.gov To: mcclanahantc@ornl.gov Oak Ridge National Laboratory. July 212014.

[50] G. E. Granroth, D. H. Vandergriff, and S. E. Nagler, "SEQUOIA: A fine resolution chopper spectrometer at the SNS," Physica B: Condensed Matter, vol. 385-386, Part 2, pp. 1104-1106, Nov. 152006.

[51] G. E. Granroth, A. I. Kolesnikov, T. E. Sherline, J. P. Clancy, K. A. Ross, J. P. C. Ruff, et al., "SEQUOIA: A Newly Operating Chopper Spectrometer at the SNS," Journal of Physics: Conference Series, vol. 251, p. 012058, 2010.

[52] E. B. Iverson, P. D. Ferguson, F. X. Gallmeier, and B. D. Murphy, "The Spallation Neutron Source high power target station moderator performance: calculations and studies," Journal of Neutron Research, vol. 11, pp. 83-91, 2003.

[53] L. DeBeer-Schmitt. Email. From: debeerschmlm@ornl.gov To: mcclanahantc@ornl.gov Oak Ridge National Laboratory. June 242014.

[54] D. L. Abernathy, M. B. Stone, M. J. Loguillo, M. S. Lucas, O. Delaire, X. Tang, et al., "Design and operation of the wide angular-range chopper spectrometer ARCS at the Spallation Neutron Source," Review of Scientific Instruments, vol. 83, pp. -, 2012.

[55] D. L. Abernathy. Email. From: abernathydl@ornl.gov To: mcclanahantc@ornl.gov Oak Ridge National Laboratory. Nov. 262014.

[56] D. L. Abernathy. Email. From: abernathydl@ornl.gov To: mcclanahantc@ornl.gov Oak Ridge National Laboratory. Dec. 102014. 